\title{
Wirkungsmonitoring der FFG Förderung 2018
}

Wien, Juni 2019

www.kmuforschung.ac.at 
Diese Studie wurde im Auftrag der Österreichischen Forschungsförderungsgesellschaft (FFG) durchgeführt.

KMU Forschung Austria

Autorinnen und Autoren

Peter Kaufmann

Elisabeth Nindl

Administration der Befragung und Layout: Mira Reisinger

Die vorliegende Studie wurde nach allen Maßstäben der Sorgfalt erstellt.

Die KMU Forschung Austria übernimmt jedoch keine Haftung für Schäden oder Folgeschäden, die auf diese Studie oder auf mögliche fehlerhafte Angaben zurückgehen.
Dieses Werk ist urheberrechtlich geschützt. Jede Art von Nachdruck, Vervielfältigung, Verbreitung, Wiedergabe, Übersetzung oder Einspeicherung und Verwendung in Datenverarbeitungssystemen, und sei es auch nur auszugsweise, ist nur mit ausdrücklicher Zustimmung des Auftraggebers der Studie gestattet.
Für Rückfragen zur Studie

Peter Kaufmann

Tel.: +4315059761 - 31

p.kaufmann@kmuforschung.ac.at

www.kmuforschung.ac.at
Mitglied bei:

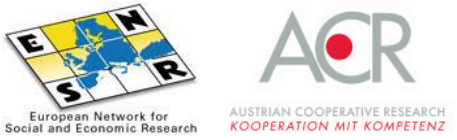




\section{Inhaltsverzeichnis}

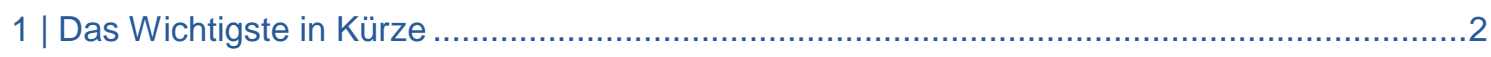

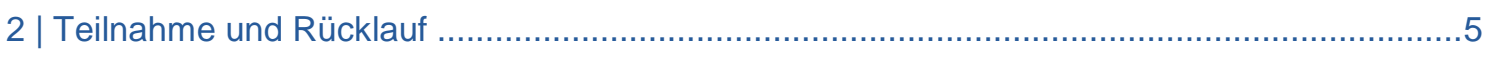

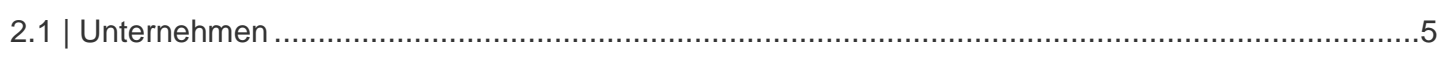

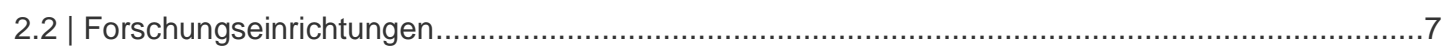

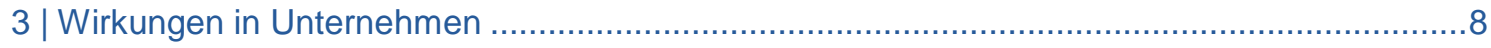

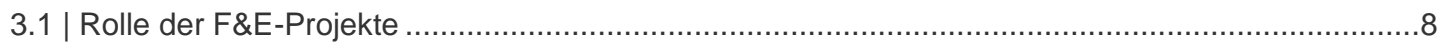

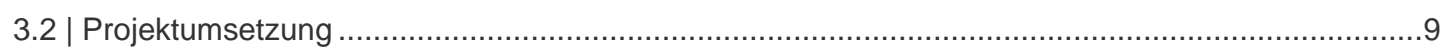

3.3 | Projektergebnisse und deren wirtschaftliche Verwertung ................................................10

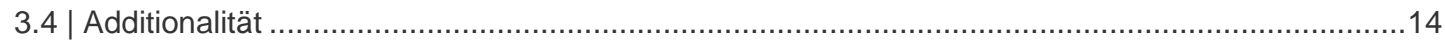

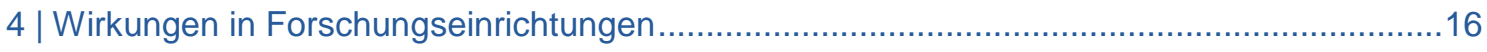

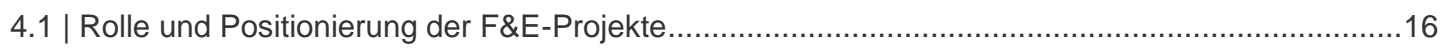

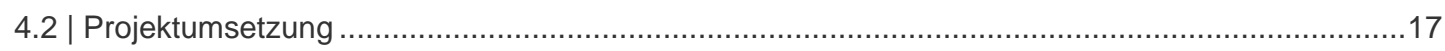

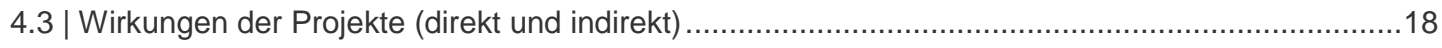

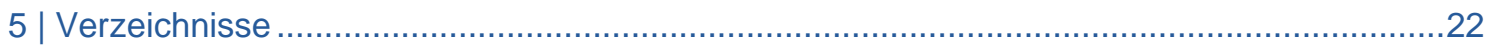




\section{1 | Das Wichtigste in Kürze}

Das Wirkungsmonitoring 2018 bezieht sich auf im Jahr 2014 beendete Forschungsprojekte. Es wurden alle Forschungsprojekte ab einer Mindestgröße auf Teilnehmerebene ausgewählt, für die kein anderwärtiges, programmspezifisches Monitoring in der FFG besteht.

Wie auch in den vergangenen Jahren konnte eine hohe Beteiligung an der Unternehmensbefragung erreicht werden. Durch die Aktualisierung der Kontaktdaten und die Versendung der Online-Befragung mit zweimaliger Erinnerung konnten $97,4 \%$ aller geförderten Unternehmen erreicht werden; der Netto-Rücklauf beträgt 66,5\% (390 Projektteilnahmen). Diese verteilen sich zu $50 \%$ auf den Bereich Basisprogramme (2017: $47 \%$ ), zu $42 \%(36 \%)$ auf die Thematischen Programme und zu $8 \%(17 \%)$ auf die Strukturprogramme. ${ }^{1}$

Das Monitoring bei Forschungseinrichtungen (FE) wurde ebenfalls mittels eines Online-Fragebogens umgesetzt. Von den 331 erreichten Projektteilnehmern beantworteten 210 den Fragebogen; dies entspricht einem Netto-Rücklauf von 63,4 \%. Der Schwerpunkt der Befragung liegt nach der Instrumentenlogik der FFG bei Kooperationsprojekten (64\%) und dem Wissenschaftstransfer (BRIDGE, $14 \%$ ). Einzelprojekte spielen eine untergeordnete Rolle, da FE im FFG Portfolio nur in Ausnahmefällen alleine Projekte durchführen.

\section{Wirkungen bei Unternehmen}

Für $9 \%$ der Unternehmen stellt das geförderte Projekt die erste F\&E-Tätigkeit im Unternehmen dar, ein leichter Rückgang im Vergleich zu den vergangenen Jahren. Für $74 \%$ der erstmalig F\&E betreibenden Unternehmen wirkte das Projekt als Anstoß für weitere F\&E-Projekte und trug somit zur Verbreiterung der Innovationsbasis bei.

$13 \%$ der Projektebeteiligung wurden unter weiblicher Projektleitung durchgeführt. Somit setzt sich der langsame Anstieg von Projektleiterinnen im Zeitverlauf auf niedrigem Niveau fort - seit 2014 kam es zu einem Zuwachs von $5 \%$-Punkten. Wenn eine Frau die Projektleitung übernimmt, dann sind signifikant mehr Frauen in den Projektteams vertreten.
Der Anteil an Projekten in bestehenden bzw. neuen Aktivitätsfeldern liegt mit $64 \%$ und $35 \%$ im Durchschnitt seit 2011. Insbesondere Unternehmen in Branchen der Mittelniedrigtechnologie sowie der Hochtechnologie betreten durch die FFG-Projekte neue Aktivitätsfelder.

Bei KU sind die Projekte häufiger Anstoß für Neues als bei MU und GU, aber auch GU stoßen immer öfter in neue Aktivitätsfelder vor. Dies geschieht besonders häufig in den Programmen COIN, ENERGIE DER ZUKUNFT und Neue Energien 2020.

Aus technischer Sicht wurden in 94 \% der Fälle die Projektziele zumindest derart erreicht, dass die Ergebnisse nutzenstiftend eingesetzt werden konnten. In $65 \%$ der Fälle konnten die Unternehmen auch die wirtschaftlichen Projektziele erreichen.

Insbesondere Großunternehmen erreichen die mit dem F\&E-Projekt verbundenen wirtschaftlichen Ziele, was sich an einer Zustimmung von $70 \%$ zeigt. Bei Kleinunternehmen und mittleren Unternehmen liegen die Werte im Bereich des langjährigen Mittels von $59 \%$. Bei Kleinunternehmen sind es insbesondere weiterführende Entwicklungsarbeiten, bei mittleren Unternehmen Veränderung der Nachfrage/des Marktpreises, die die wirtschaftliche Zielerreichung hemmen. Bei den weiterführenden Entwicklungsarbeiten ist ein Anstieg über die Jahre zu verzeichnen, der auf Projekte in den Kooperationsformaten zurückzuführen ist. Das bestätigt, dass diese Projekte in anwendungsferneren Entwicklungsstufen realisiert werden. Dies steht in Einklang damit, dass heuer mehr Befragte angaben, die Fragestellungen in F\&E-Folgeprojekten fortzusetzen.

Bei Projektbeteiligungen mit wirtschaftlichem Verwertungsziel konnten in den ersten vier Jahren nach Projektende in $58 \%$ der Fälle Arbeitsplätze durch die Verwertung der Projektergebnisse gesichert oder geschaffen werden (2011-2014 rd. 65\%, 2015-2018 rd. 59\%) wobei hier die konjunkturelle Entwicklung maßgeblichen Einfluss nimmt.

$21 \%$ der geschaffenen bzw. gesicherten Arbeitsplätze entfallen auf Kleinunternehmen (2017: $29 \%$ ), der Anteil von Großunternehmen ist wieder von $63 \%$ auf $70 \%$

\footnotetext{
1 Für eine Übersicht über die Programme und deren Zuordnung zu Förderinstrumenten siehe Tabelle $10 \mathrm{im}$ Anhang.
} 
angestiegen, jener von mittleren Unternehmen liegt relativ konstant bei rd. $8 \%$. Der aktuelle Rückgang bei Kleinunternehmen ist durch geringere Beschäftigungswirkungen in den kleinen Unternehmen der Hochtechnologie begründet.

Direkte Beschäftigungseffekte bei F\&E-Mitarbeiter_innen entstanden in $73 \%$ der Projektbeteiligungen. $64 \%$ der neuen F\&E-Stellen wurden während der Projektlaufzeit geschaffen. $53 \%$ davon wurden durch unternehmensinterne Umschichtungen besetzt, $47 \%$ wurden extern rekrutiert. Im Unterschied dazu entfallen $68 \%$ der nach Projektende geschaffenen F\&E-Positionen auf extern rekrutierte Personen. Beim Instrument Einzelprojekte wurde sowohl während dem Projekt, als auch danach häufiger extern F\&E-Personal rekrutiert als intern umgeschichtet. Wie auch 2017 wurden bei Kooperationsprojekten mehr externe F\&E-Mitarbeiter_innen nach Projektende als während dem Projekt eingestellt, was auf verstärkten weiteren F\&E-Bedarf deutet.

In $75 \%$ der Projektbeteiligungen trat eine Verbesserung der technologischen Wettbewerbsposition ein (2017: 69 \%). Innerhalb dessen wurde nach Einschätzung der Unternehmen bei $11 \%$ der Projektteilnahmen zur internationalen Spitze aufgeschlossen, was besonders auf mittlere Unternehmen zutrifft; instrumentenspezifische Unterschiede hinsichtlich der technologischen Wettbewerbsposition sind jedoch - wie bei allen Verwertungsaspekten - stark ausgeprägt.

Aus $94 \%$ der Projektteilnahmen resultierte zumindest eine innovative Teillösung. Die Arten der Innovationen verteilen sich über die Jahre weitgehend konstant: der Anteil von neuen Produkten hat sich wieder leicht auf $29 \%$ erhöht, jener von veränderten Produkten auf $17 \%$; diese Verschiebung geht zulasten von neuen Dienstleistungen, die nun bei 8,5 \% liegen.

In dieser Befragung wurde erstmals erhoben, inwiefern das geförderte F\&E-Projekt einen Einfluss auf ein potenziell neues Geschäftsmodell hatte. Aggregiert war dies in rd. $6 \%$ der Fall, vor allem bei mittleren Unternehmen (11\%), während dies bei kleinen sowie großen Unternehmen kaum zutraf (4\%).

Die wirtschaftliche Verwertung der Ergebnisse erfolgte in $50 \%$ der Projektbeteiligungen innerhalb von vier Jahren nach Projektende. Große und mittlere Unternehmen weisen hier Anteile von 56 \% bzw. 54 \% auf, während in Kleinunternehmen nur $45 \%$ die Projektergebnisse zum Zeitpunkt der Befragung bereits verwerten. In Einzelprojekten verwerten im Jahr 2018 bereits $65 \%$ der
Unternehmen die Projektergebnisse, bei Kooperationsprojekten sind es $33 \%$. Unternehmen im Dienstleistungsbereich verwerten später als Unternehmen in der Herstellung von Waren.

Schutzrechte wurden in $27 \%$ der untersuchten Projekte (2017: $25 \%$ ) angemeldet. Besonders bei Einzelprojekten, sowie in den Branchen der Hochtechnologie, Mittelhochtechnologie oder auch Niedrigtechnologie meldeten Unternehmen Schutzrechte an. Auch 2018 versuchen einige Unternehmen mit einer hohen Anzahl von Patenten eine technologische Nische strategisch abzudecken.

Neben den untersuchten Förderprogrammen haben 39 \% der Unternehmen auf zusätzliche Förderangebote für das Forschungsthema zurückgegriffen. In $62 \%$ der Fälle wurde eine nationale Förderung genannt, in $21 \%$ eine Förderung auf EU-Ebene und in $16 \%$ eine regionale Förderung. Somit vergrößert sich der Vorsprung von europäischen zu regionalen Förderungen, der erstmals 2017 auftrat.

Die F\&E-Aktivitäten trugen dazu bei, dass in $75 \%$ der Projektteilnahmen neue Kontakte etabliert bzw. bestehende Kontakte intensiviert wurden (2017: 80 \%). Mittlere und große Unternehmen knüpfen häufiger Kontakte entlang der Wertschöpfungskette, als Kleinunternehmen. Nach Technologieintensitäten zeigt sich, dass mit einer höheren Technologie- bzw. Wissensintensität die Interaktion steigt: im Bereich der Hochtechnologie berichten $93 \%$ der Unternehmen von neuen/intensivierten Kontakten, in jenem der Niedrigtechnologie nur $54 \%$; bei den wissensintensiven Dienstleistungen liegt dieser Wert bei $74 \%$ (62 \% bei den weniger wissensintensiven).

$64 \%$ der Unternehmen tätigten Zusatzinvestitionen, um die Projektergebnisse auf dem Markt zu platzieren. Im Median tätigte ein KU zusätzliche Investitionen in Höhe von $€ 110.000$, ein $\mathrm{MU}$ von $€ 200.000$ und ein $\mathrm{GU}$ von $€ 500.000$.

Insgesamt wären $39 \%$ der Projekte ohne Förderung nicht durchgeführt worden (2017: $32 \%$ ), weitere $51 \%$ wären nur in geringerem Ausmaß umgesetzt worden (2017: $57 \%$ ) und $89 \%$ nur mit zeitlicher Verzögerung (2017: $92 \%$ ). Besonders häufig wären Innovationsnetzwerke $(69 \%)$ und Kooperationsprojekte (56\%) nicht umgesetzt worden, die somit eine höhere Additionalität der Förderung aufweisen. Die Unterschiede zu den Einzelprojekten (23\%) sind primär durch die jeweilige Instrumentenlogik sowie die höheren Fördersätze begründbar. Zudem zeigt sich auch 2018 wieder, dass 
kleinere Unternehmen geringere Fähigkeiten/ Bereitschaften aufweisen, Projekte ohne Fördermittel durchzuführen.

\section{Wirkungen bei Forschungseinrichtungen}

Im Gegensatz zum Anstieg in den vergangenen Jahren ist der Anteil an Projekten, die in bestehende thematische Schwerpunkte der Forschungseinrichtungen fallen, leicht auf 59\% gesunken (2017: $62 \%$ ). Analog dazu ist der Anteil jener Projekte, die der FE als Grundlage eines neuen Schwerpunktes dienen, von $28 \%$ auf $32 \%$ gestiegen (insbesondere in den Programmen Bridge (39\%), COIN (53\%) und Neue Energien 2020 (38\%)). In den übrigen Fällen streifte das Projekt Randbereiche der Forschungsaktivitäten am Institut.

Für die Umsetzung der F\&E-Projekte wurden von den FE 1.088 Mitarbeiter_innen eingesetzt - damit waren an einem Projekt durchschnittlich 5,4 Personen beteiligt. Die nach der Zahl der Mitarbeiter_innen gemessen größten Projekte wurden von Fachhochschulen abgewickelt $(8,1)$, gefolgt von Universitäten $(5,5)$ und außeruniversitären FE $(5,1)$.

Rund $34 \%$ aller Projektmitarbeiter_innen waren Diplomand_innen (inkl. Bachelor- und Masterstudierende), $30 \%$ sonstiges Forschungspersonal (Laborpersonal, aber auch Professor_innen oder Dozent_innen), $22 \%$ stellen Doktoratsstudent_innen und $14 \%$ Post-Docs. In $77 \%$ der Projektteilnahmen wurden Diplomand_innen und/oder Doktorand_innen eingesetzt, und in $60 \%$ Post Docs.

Rund $65 \%$ der Forschungseinrichtungen konnten die eigenen Projektziele in vollem Umfang umsetzen, weitere $31 \%$ zum größten Teil. Dies stellt eine deutliche Verschiebung von $10 \%$-Punkten zugunsten der vollständigen Zielerreichung dar und entspricht Werten, die nur 2014 erreicht wurden.

Ähnliches gilt für die Einschätzungen zum Gesamtprojekt: Bei $49 \%$ der Projekte (2017: $40 \%$ ) wurden auch die Ziele des Gesamtprojektes in vollem Umfang erreicht, in weiteren $45 \%$ "trifft [dies] ziemlich zu“ (2017: $55 \%)$. Zusammengenommen deckt sich dies mit den
Angaben der Unternehmen, die zu $94 \%$ die technischen Projektziele zumindest teilweise erreichten.

Eine zukünftige Zusammenarbeit mit den wissenschaftlichen Partnern nach einer erstmaligen Kooperation planen $26 \%$ der FE (2017: $27 \%$ ), nach einer erstmaligen Zusammenarbeit mit Unternehmen sind es $37 \%$ (2017: $39 \%$ ). Bei längerfristigen Zusammenarbeiten liegt dieser Anteil deutlich höher - die Kooperation mit FE/Unternehmen wollen $58 \%$ bzw. $67 \%$ fortführen.

Der Anstoß von Abschlussarbeiten ist eine wichtige Wirkungskategorie bei FE: In $58 \%$ der Teilnahmen führten die Projekte zu insgesamt 301 weiterführenden Diplomarbeiten oder Dissertationen (durchschnittlich 1,9). Der Wechsel von F\&E-Personal zu Unternehmenspartnern des Projekts erfolgte in $15 \%$ der Fälle (2017: $16 \%$ ).

Disseminationsaktivitäten erfolgen primär mit Beteiligung der Projektpartner. Wie bereits in den Vorjahren ist die Publikation der Projektergebnisse in akademisch referierten Journalen die häufigste Form der Dissemination mit $81 \%$ der Projekte. Insgesamt wurden 1.531 Transferaktivitäten gesetzt (2017: 1.550), im Durchschnitt 7,3 je Projektteilnahme (Vorjahr: 6,7).

Zu geförderten Folgeprojekten kam es in $60 \%$ der Projektbeteiligungen (Vorjahre: $57 \%$ bis $63 \%$ ). Daraus entstanden mindestens 282 Folgeprojekte - im Durchschnitt folgten 2,3 Projekte auf das FFG-Projekt. Die wichtigste Finanzierungsquelle ist wiederum die FFG mit $45 \%$ (Vorjahre: $42 \%$ bis $49 \%$ ). EU-Folgeprojekte ergaben sich bislang in $10 \%$ der Projektteilnahmen (Vorjahre: $9 \%$ bis $14 \%$ ), direkt durch Unternehmen und Institutionen finanzierte Folgeprojekte liegen bei $21 \%$ (Vorjahre: $20 \%$ bis $21 \%$ ).

Schließlich deutet die relativ hohe Zustimmung zur potenziellen Nutzung der Projektergebnisse in anderen Anwendungsfeldern auf Möglichkeiten zum Technologietransfer hin, die einer gesonderten Untersuchung bzw. eine erhöhte Aufmerksamkeit seitens der Politik bedürfen könnte. 


\section{2 | Teilnahme und Rücklauf}

\subsection{Unternehmen}

Für im Jahr 2014 abgeschlossene Projekte wurden von der FFG 789 Projektbeteiligungen von Unternehmen an die KMU Forschung Austria übermittelt. Nach der Bereinigung der Daten ${ }^{2}$ wurden 604 Unternehmen per Email kontaktiert. Rückmeldungen kamen zu 390 Projektbeteiligungen von bestehenden, inländischen Unternehmen (zweimalige elektronische Erinnerung). Durch die ex-ante Aktualisierung der Kontaktdaten (postalisches Anschreiben) konnten $98 \%$ der Unternehmen per Email erreicht werden.

Tabelle 1 Versendung und Rücklauf: Unternehmen nach Bereichen und Programmen

\begin{tabular}{|c|c|c|c|}
\hline Programm & Erreicht & Ausgewertet & Rücklauf \\
\hline \multicolumn{4}{|c|}{ Basisprogramme (BP) } \\
\hline BASIS & 262 & 181 & $69 \%$ \\
\hline Bridge & 19 & 11 & $58 \%$ \\
\hline EUROSTARS & 4 & 2 & $50 \%$ \\
\hline \multicolumn{4}{|c|}{ Thematische Programme (TP) } \\
\hline Neue Energien 2020 & 83 & 57 & $69 \%$ \\
\hline IV2Splus & 44 & 30 & $68 \%$ \\
\hline FIT-IT & 29 & 19 & $66 \%$ \\
\hline Leuchttürme eMobilität & 23 & 14 & $61 \%$ \\
\hline Produktion der Zukunft & 18 & 9 & $50 \%$ \\
\hline benefit & 15 & 11 & $73 \%$ \\
\hline ENERGIE DER ZUKUNFT & 15 & 8 & $53 \%$ \\
\hline TAKE OFF & 14 & 7 & $50 \%$ \\
\hline AT:net & 8 & 5 & $63 \%$ \\
\hline NANO & 8 & 4 & $50 \%$ \\
\hline e!MISSION & 1 & 1 & $100 \%$ \\
\hline \multicolumn{4}{|c|}{ Strukturprogramme (SP) } \\
\hline COIN & 34 & 19 & $56 \%$ \\
\hline Research Studios Austria & 1 & 1 & $100 \%$ \\
\hline \multicolumn{4}{|c|}{ Agentur für Luft- und Raumfahrt (ALR) } \\
\hline ASAP & 13 & 11 & $85 \%$ \\
\hline Gesamt & 591 & 390 & $66,5 \%$ \\
\hline
\end{tabular}

Quelle: KMU Forschung Austria

Die 591 erreichten Projektteilnehmer_innen verteilen sich auf 452 geförderte Projekte; die 390 ausgewerteten Fragebögen auf 324 Projekte; d.h. dass bei $72 \%$ der geförderten Projekte zumindest ein Fragebogen ausgefüllt wurde.

Die Unternehmen beantworten den Fragebogen aus ihrer spezifischen Sicht des eigenen Projektteils, weshalb die Auswertungen durchgehend auf Ebene der Projektbeteiligung erfolgt.

\section{Weniger Projekte aus den Strukturprogrammen}

Das Basisprogramm stellt mit $46 \%$ (2017: $42 \%$ ) der Projektbeteiligungen nach wie vor den größten Anteil im FFG Wirkungsmonitoring (WiMon). Der im Vergleich höhere Anteil des Basisprogramms ergibt sich aus einer geringeren Anzahl von Projekten im Bereich der Strukturprogramme $(5,1 \%$ gegenüber $12 \% 2017)$. So wurden 2014 nur 19 COIN-Netzwerk Projektbeteiligungen erfasst - 2017 waren es noch 43. Der Anteil der Thematischen Programme liegt bei $43 \%$ bzw. 165 Projektteilnahmen. Auf das Programm Neue Energien 2020 entfallen $15 \%$ der Projektbeteiligungen, auf IV2Splus $8 \%$ und auf FIT-IT $5 \%$.

Nach der Logik der Förderinstrumente ist der höchste Anteil mit $47 \%$ der Projektteilnahmen den Einzelprojekten zuzuordnen; auf die Kooperationsprojekte entfallen $38 \%$. Für diese ist eine hohe Programmheterogenität charakteristisch - Kooperationsprojekte können 12 Programmen zugeordnet werden. Insgesamt umfasst die diesjährige Auswertung 11 Instrumente in 17 Programmen mit 24 Programmlinien.

\section{$22 \%$ der Unternehmen mit bis zu 10 Beschäftigten, $14 \%$ über 1.000}

Wie auch in den Vorjahren stellen Projektbeteiligungen von Unternehmen mit 1 bis 20 Beschäftigten (32\%) den größten Anteil im WiMon. Von jenen 119 Projektteilnahmen entfallen 79 auf Kleinstunternehmen mit bis zu 10 Beschäftigten, ein Rückgang gegenüber 2017 um 2 \%Punkte. Umgekehrt stieg der Anteil von Unternehmen mit 11 bis 20 Personen leicht auf $10,7 \%$. 


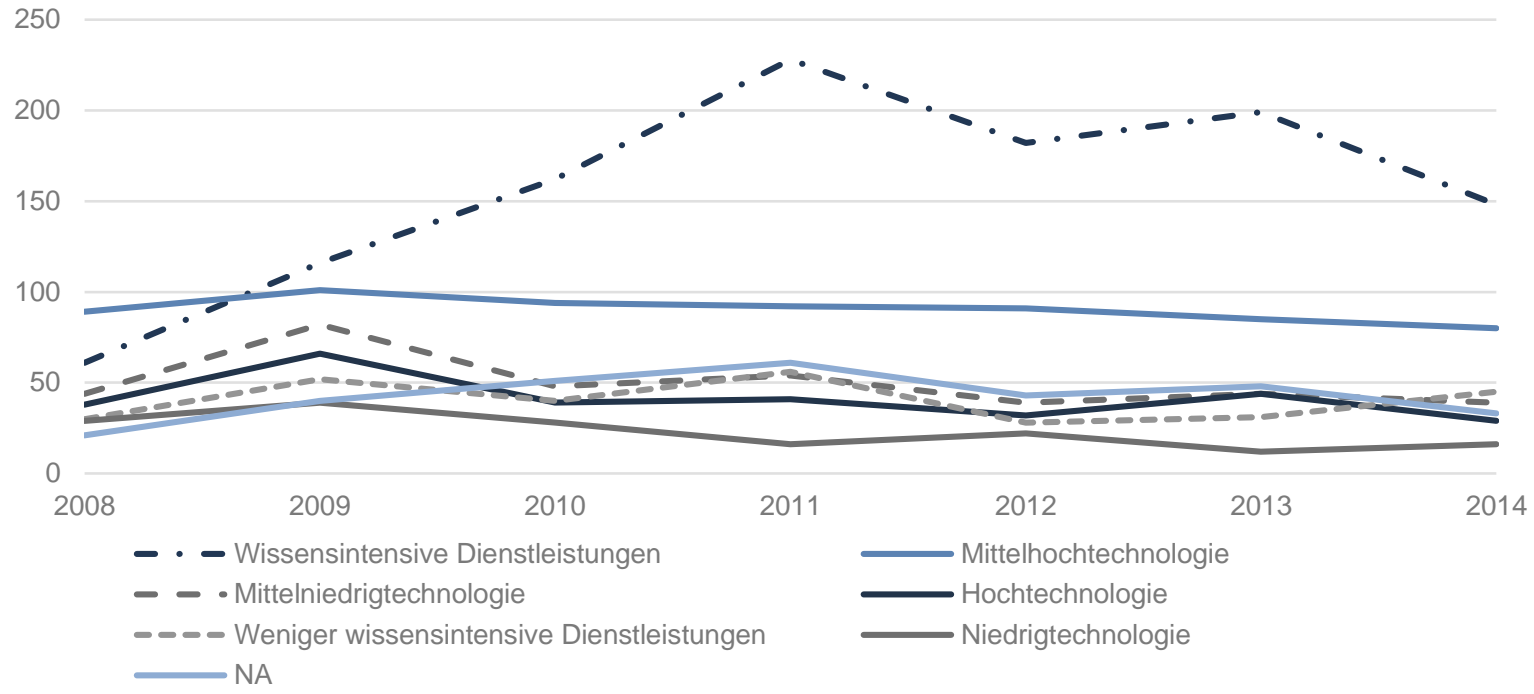

Quelle: KMU Forschung Austria

NA...Projekte in nicht nach Technologiefeldern/Wissensintensität zuordenbare Wirtschaftszweige.

Die zweitgrößte Gruppe bilden die Unternehmen zwischen 21 und 50 Beschäftigten mit 17\%, ebenfalls ein leichter Zuwachs gegenüber 2017. Ein Rückgang der Teilnahme ist bei Unternehmen mit 101 bis $250 \mathrm{Be}$ schäftigten zu beobachten (10,7\% vs. $12,8 \% 2017)$; die Projektbeteiligungen von Unternehmen über 1.000 Beschäftigte belaufen sich wie 2017 auf $14 \%$. Dies entspricht 50 Projektbeteiligungen von 19 verschiedenen Unternehmen.

Nach der Klassifikation der Europäischen Union wurden, wie auch 2017, 45 \% aller betrachteten Projektteilnahmen von Kleinunternehmen (KU) durchgeführt, $12 \%$ von mittleren Unternehmen (MU) und $43 \%$ von Großunternehmen (GU).

\section{Hoher Anteil wissensintensiver Dienstleistungen}

Forschungsvorhaben, die Unternehmen aus dem ÖNACE Abschnitt Herstellung von Waren zurechenbar sind, stellen wie auch 2017 mit $40 \%$ den größten Anteil an FFG-geförderten Projekten. Der Anteil an Projektbeteiligungen von Unternehmen aus Dienstleistungsbranchen (Abschnitte J, K, L, M und N) beträgt $43 \%$ aller Projektteilnahmen (2017: $48 \%$ ).

Der Anteil der wissensintensiven Dienstleistungen ist gegenüber 2017 von $43 \%$ auf $38 \%$ gesunken, während jener der weniger wissensintensiven von $7 \%$ auf rd. $12 \%$ zugenommen hat. Auch in Herstellung von Waren kam es zu einer leichten Verschiebung in Richtung geringerer Technologieintensitäten. Eine mögliche Erklärung dafür liegt in mehr freien Kapazitäten für F\&E auch in Unternehmen in weniger wissensintensiven Bereichen aufgrund der wirtschaftlichen Stagnation in den Antragsjahren 2010-2013.

Kleinunternehmen sind primär im Bereich der wissensintensiven sowie weniger wissensintensiven Dienstleistungen vertreten, relativ gesehen stellen sie aber auch den größten Anteil im Bereich der Hochtechnologie.

Abbildung 2 Technologieintensität nach Unternehmensgröße

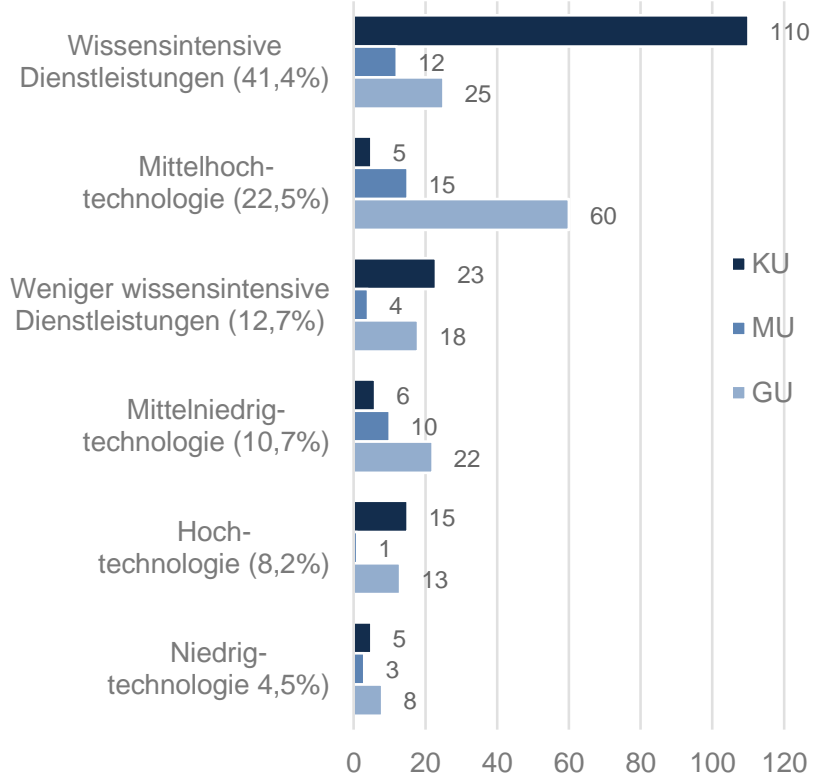

Quelle: KMU Forschung Austria; $\mathrm{n}=390$

Wissensintensive Kleinunternehmen sind am stärksten im Bereich Architektur- und Ingenieurbüros sowie technische, physikalische und chemische Untersuchung und der Erbringung von Dienstleistungen der Informationstechnologie vertreten, sowie im Bereich Forschung und Entwicklung allgemein. Die $15 \mathrm{KU}$ im Bereich Hochtechnologie sind alle in der Branche Herstellung von 
Datenverarbeitungsgeräten, elektronischen und optischen Erzeugnissen aktiv. Im Unterschied dazu sind die GU im WiMon primär in der Mittelhochtechnologie tätig, insbesondere in der Herstellung von elektronischen Ausrüstungen und dem Maschinenbau. 13 Projektbeteiligungen von GU sind der Herstellung von Datenverarbeitungsgeräten, elektronischen und optischen Erzeugnissen zuzuordnen, 15 Projektbeteiligungen im Bereich der Energieversorgung fallen nicht in die Klassifikation nach Technologieintensität.

Der Barwert der Förderungen der in der Befragung abgedeckten Projektbeteiligungen beläuft sich auf $€ 77$ Mio., die Gesamtkosten in den Unternehmen betrugen knapp $€ 234$ Mio. Der durchschnittliche Förderbarwert liegt wie auch im Vorjahr bei $€ 199.000$, der Median bei $€ 103.000$. Insgesamt haben sich die Volumina vergrößert: in der aktuellen Auswertung sind um $15 \%$ weniger Unternehmen als im Vorjahr enthalten, die Gesamtkosten der Projekte sind aber nur um 5 \% zurückgegangen.

Tabelle 2 Projektkosten nach Förderungs- und Finanzierungs-instrumenten sowie Unternehmensgröße in Tsd. €

\begin{tabular}{lccccccc}
\hline & n & \multicolumn{2}{c}{ Gesamtkosten } & \multicolumn{2}{c}{ Barwert } & \multicolumn{2}{c}{ Förderquote } \\
& & $\begin{array}{c}\text { Mit- } \\
\text { tel- } \\
\text { wert }\end{array}$ & $\begin{array}{c}\text { Medi } \\
\text {-an }\end{array}$ & $\begin{array}{c}\text { Mit- } \\
\text { tel- } \\
\text { wert }\end{array}$ & $\begin{array}{c}\text { Medi } \\
\text {-an }\end{array}$ & $\begin{array}{c}\text { Mittel- } \\
\text { wert }\end{array}$ & $\begin{array}{c}\text { Medi } \\
\text {-an }\end{array}$ \\
\hline EP & 213 & 752 & 453 & 223 & 140 & $33 \%$ & $29 \%$ \\
KP & 175 & 374 & 145 & 195 & 92 & $62 \%$ & $60 \%$ \\
\hline INNO & 14 & 95 & 92 & 61 & 57 & $65 \%$ & $65 \%$ \\
\hline Andere & 43 & 156 & 112 & 87 & 60 & $56 \%$ & $60 \%$ \\
\hline GU & 198 & 840 & 468 & 279 & 161 & $39 \%$ & $34 \%$ \\
\hline MU & 58 & 468 & 254 & 181 & 110 & $47 \%$ & $43 \%$ \\
\hline KU & 207 & 2574 & 145 & 127 & 76 & $56 \%$ & $53 \%$ \\
\hline
\end{tabular}

Quelle: KMU Forschung Austria; N=390. EP...Einzelprojekt, KP...Kooperationsprojekt, INNO...Innovationsnetzwerk.

\section{2 | Forschungseinrichtungen}

Zur Befragung der Forschungseinrichtungen (FE) wurden per Email Einladungen an die technischen Projektleiter_innen, und bei Nichterreichung an allgemeine Adressen (z.B. Sekretariate) von FE mit FFG-geförderten Projekten verschickt.

Von der FFG wurden Daten zu 586 Projektteilnahmen übermittelt. Projekte mit einem Volumen unter $€ 40.000$ sowie aus nicht relevanten Instrumenten (Sondierung, Dissertationen) wurden ausgeschlossen. So wurden 341 Projektteilnahmen identifiziert, wovon 331 erreicht werden konnten und 210 den Fragebogen beantworteten - dies entspricht einem Netto-Rücklauf von $63,4 \%$.

Die 210 analysierten Projektteilnahmen wurden in 171 Projekten von 121 verschiedenen FE umgesetzt.

Tabelle 3 Versendung und Rücklauf: FE nach Bereichen und Programmen

\begin{tabular}{|c|c|c|c|}
\hline Programm & Erreicht & Im Survey & Rücklauf \\
\hline \multicolumn{4}{|c|}{ Basisprogramme (BP) } \\
\hline Bridge & 48 & 29 & $67 \%$ \\
\hline EUROSTARS & 4 & 1 & $25 \%$ \\
\hline \multicolumn{4}{|c|}{ Thematische Programme (TP) } \\
\hline Neue Energien 2020 & 69 & 47 & $68 \%$ \\
\hline IV2Splus & 36 & 22 & $61 \%$ \\
\hline FIT-IT & 31 & 20 & $65 \%$ \\
\hline NANO & 19 & 9 & $47 \%$ \\
\hline benefit & 16 & 11 & $69 \%$ \\
\hline Produktion der Zukunft & 15 & 11 & $73 \%$ \\
\hline TAKE OFF & 14 & 9 & $64 \%$ \\
\hline ENERGIE DER ZUKUNFT & 13 & 7 & $54 \%$ \\
\hline Leuchttürme eMobilität & 5 & 5 & $100 \%$ \\
\hline Energieforschung (e!MISSION) & 2 & 1 & $50 \%$ \\
\hline \multicolumn{4}{|c|}{ Strukturprogramme (SP) } \\
\hline COIN & 28 & 20 & $71 \%$ \\
\hline Research Studios Austria & 15 & 7 & $47 \%$ \\
\hline wfForte & 4 & 2 & $50 \%$ \\
\hline FEMtech & 2 & 2 & $100 \%$ \\
\hline AplusB & 1 & 1 & $100 \%$ \\
\hline \multicolumn{4}{|c|}{ Agentur für Luft- und Raumfahrt (ALR) } \\
\hline ASAP & 9 & 6 & $67 \%$ \\
\hline Gesamt & 331 & 210 & $63,4 \%$ \\
\hline
\end{tabular}

Quelle: KMU Forschung Austria

\section{Breite Streuung über die Programme}

Auch 2018 liegt der Schwerpunkt bei den Thematischen Programmen (68\%, 2017: 61\%), gefolgt von den Strukturprogrammen (15\%, 2017: $20 \%)$ und dem Bereich Basisprogramm (17\%, $13 \%$-Punkte davon entfallen auf Bridge) und ALR (3\%).

Die Schwerpunktprogramme bilden wie auch im WiMon 2017 Neue Energien 2020, IVS2plus, FIT-IT, Bridge und COIN mit insgesamt 138 Förderfällen bzw. $66 \%$ der rückmeldenden Projektteilnahmen.

Nach Organisationstypus liegt der Fokus der 2014 abgeschlossenen Projekte bei Universitäten mit $46 \%$ der Fragebögen (2017: $53 \%$ ). Außeruniversitäre Forschungseinrichtungen (AUF) sind wie im Vorjahr mit rd. $34 \%$ vertreten, der Anteil der Fachhochschulen (FH) stieg von $6 \%$ auf $8 \%$. Kompetenzzentren sind mit 17 Fällen gegenüber neun im Vorjahr stärker präsent. 


\subsection{Rolle der F\&E-Projekte}

\section{Beitrag zur Verbreiterung der Innovationsbasis: F\&E-Neueinsteiger führen in $74 \%$ der Fälle erneut F\&E-Aktivitäten durch}

Die Frage nach der Fortführung bestehender F\&E-Tätigkeiten gegenüber dem Einstieg in $\mathrm{F} \& \mathrm{E}$ erweist sich als im Zeitverlauf stabil. In $88 \%$ der Projektteilnahmen wurden bestehende F\&E-Aktivitäten ausgebaut, in $9 \%$ stellte das Projekt die erste F\&E-Aktivität dar, für rd. $3 \%$ blieb es auch die einzige. Somit betreiben etwa $74 \%$ der FuE-Einsteiger in Folge des geförderten Projekts weitere FuE-Aktivitäten (2011-2017: 76 \%).

Abbildung 3 Rolle des FFG-Projekts, Projektende 2007-2014

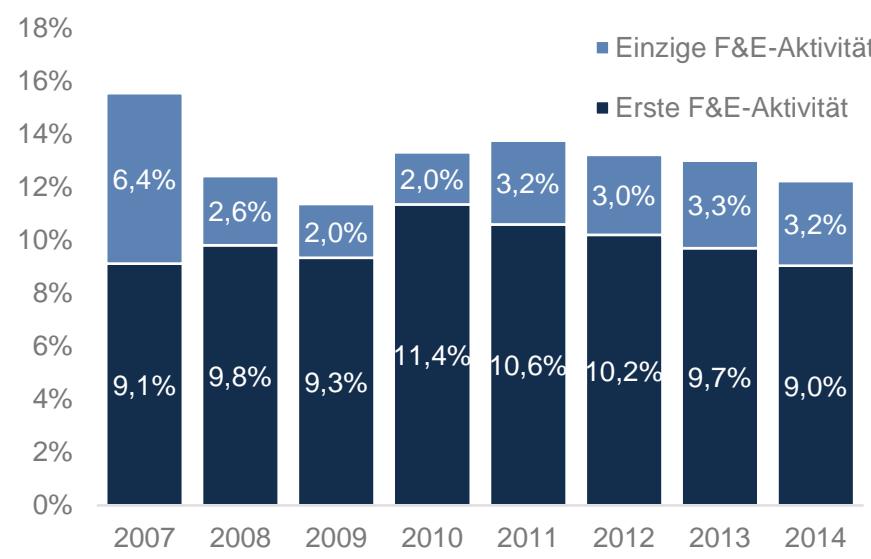

Quelle: KMU Forschung Austria. Rest auf $100 \%$ entfällt auf die Kategorie „Durch das Projekt wurden bestehende F\&E-Aktivitäten weitergeführt/ausgebaut".

In kleinen Unternehmen war die Projektteilnahme in $14 \%$ der Fälle die erste F\&E-Aktivität. Mittleren Unternehmen hingegen dienen die Projekte in $98 \%$ der Fälle dem Ausbau der Aktivitäten. Auch einige Großunternehmen werten das geförderte Projekt als erste F\&EAktivität - dies bezieht sich vermutlich aber auf den spezifischen Bereich, als auf das gesamte Unternehmen.

\section{Projekte von Kleinunternehmen wieder vermehrt in bestehenden Aktivitätsfeldern}

Entgegen dem seit 2011 zu beobachtenden Trend eines zunehmenden Anteils von Projekten in für das Unternehmen neuen Aktivitätsfeldern ist 2018 der Anteil an Projekten in bestehenden Bereichen auf 64,2 \% gestiegen, ein Wert der zuletzt 2012 erreicht wurde. Umgekehrt stellte das geförderte Projekt für nur $35 \%$ einen
Anstoß für ein neues Aktivitätsfeld dar. Diese Verschiebung ist durch die Projektbeteiligungen von KU begründet, deren Projekte 2017 nur zu $54 \%$ in bestehenden Feldern lagen, 2018 allerdings zu $63 \%$. Somit nähern sich $\mathrm{KU}$ den Werten von mittleren und großen Unternehmen an.

Die Verschiebung zwischen den Aktivitätsfeldern findet in allen Förderinstrumenten statt und selbst in den Innovationsnetzwerken wurden $67 \%$ der Projektbeteiligungen in bestehenden Forschungsbereichen verortet.

\section{$75 \%$ der Projektthemen fließen in F\&E-Folgepro-} jekte ein

Über alle Unternehmensgrößen hinweg ist 2018 ein Anstieg des Anteils jener Projektbeteiligungen zu verzeichnen, die von den bearbeitenden Unternehmen in Folgeprojekten weiterverfolgt werden. Insgesamt treiben $75 \%$ (2011-2017: $71 \%)$ der Unternehmen die Projektthemen in F\&E-Folgeprojekten voran (KU: $74 \%$, MU: $71 \%$, GU: $78 \%$ ).

Wie auch im Vorjahr resultierten Einzelprojekte (78\%) am häufigsten in Folgeaktivitäten, bei Kooperationsprojekten und Innovationsnetzwerken wird aber ein Anstieg beobachtet: bei Kooperationsprojekten wurde in $73 \%$ der Fälle das Thema weiterverfolgt (2017: $66 \%$ ), in Innovationsnetzwerken zu 63 \% (2017: $49 \%$ ). Im Instrument Wissenschaftstransfer liegt der Wert unverändert bei $63 \%$.

\section{Weitere Förderangebote werden primär auf nationa- ler Ebene in Anspruch genommen}

Rund $35 \%$ der teilnehmenden Unternehmen (121 Fälle) nahmen zur weiteren Bearbeitung des Projektthemas F\&E-Förderungen in Anspruch (2017: $39 \%$ ). In $62 \%$ der Fälle wurde eine nationale Förderung genannt (2017: $58 \%$ ), in $21 \%$ eine auf EU-Ebene und in $16 \%$ eine regionale Förderung. Somit setzt sich die im Vorjahr erstmals eingetretene Situation fort, dass häufiger EU-Förderungen als regionale in Anspruch genommen wurden (2017: EU $22 \%$, regional $19 \%$ ).

In $30 \%$ der Fälle wurden von Unternehmen für weitere Arbeiten (z. B. weiterführende F\&E-Projekte) zum jeweiligen Projektthema Förderungen auf zwei Ebenen in Anspruch genommen (2017: $22 \%)$. Dies impliziert, dass sich die Förderungen auf weniger Unternehmen konzentrieren, was sich insbesondere bei Kleinunternehmen zeigt. 
Von den 121 Unternehmensbeteiligungen mit weiteren Förderungen nannten 116 die Förderquellen: $93 \mathrm{Mal}$ $(80 \%)$ die FFG, neun Mal (8\%) die AWS und 14 Mal durch sonstige Programme (12\%). Somit verstärkt sich die Bedeutung der FFG in der Förderkette für F\&E-Projekte von Unternehmen noch weiter (2017: 72\%). Von den 93 weiterführenden FFG-Förderungen entfallen $44 \%$ auf Einzelprojekte und $40 \%$ auf Kooperationsprojekte, sowie 15\% auf andere Instrumente (2017: $47 \%$, $38 \%, 15 \%)$.

\section{2 | Projektumsetzung}

\section{Frauen weiterhin unterrepräsentiert, auch wenn die Teams bunter werden}

Mit der Bearbeitung der geförderten F\&E-Projekte waren 4.076 F\&E-Mitarbeiter_innen befasst, was wie im Vorjahr rund elf Beschäftigten pro Projektbeteiligung entspricht. Auch wenn in $63 \%$ der Projektbeteiligungen (vgl. dazu rote Dreiecke in Abbildung 4), dem höchsten bisher erreichten Wert, Projektmitarbeiterinnen involviert waren, werden insgesamt nur $18 \%$ (695) der Positionen von Frauen besetzt (vgl. dazu dunkelblaue Rauten in Abbildung 4).

Abbildung 4 Genderverteilung Projektmitarbeiter Projektende 20072014

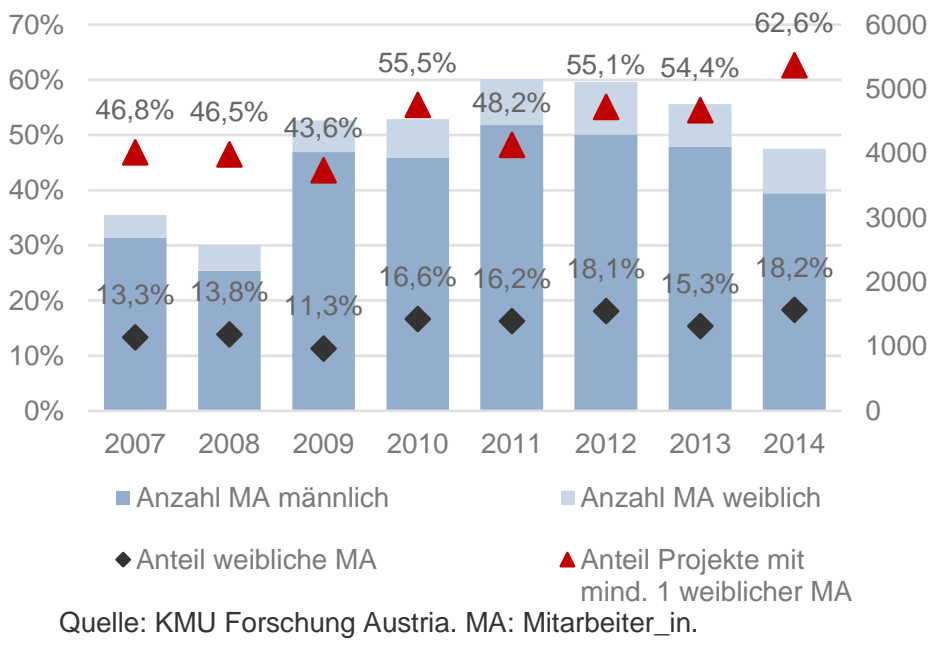

\section{Anteil der Projektleiterinnen steigt langsam}

$13 \%$ der Projektebeteiligungen wurden unter weiblicher Projektleitung umgesetzt. Damit setzt sich der langsame Anstieg von Projektleiterinnen im Zeitverlauf auf niedrigem Niveau fort, sodass es seit 2014 zu einem Zuwachs von $5 \%$-Punkten kam. Am häufigsten sind Frauen in Kleinunternehmen mit der Projektleitung betraut (15\%), gefolgt von Großunternehmen (12\%), während Projekte von Frauen in mittleren Unternehmen nur in rd. $6 \%$ der Fälle vorkommen.

Wurde jedoch ein F\&E-Projekt von einer Frau geleitet, dann waren auch deutlich häufiger und mehr Projektmitarbeiterinnen beschäftigt: in jenen 50 von Frauen geleiteten Projekten waren in $92 \%$ Frauen zur Bearbeitung der Fragestellungen beschäftigt, die in Summe $40 \%$ aller Projektmitarbeiter_innen ausmachen. In den rückmeldenden 330 von Männern geleiteten Projekten wurden in nur $52 \%$ der Fälle Frauen beschäftigt, die nur $14 \%$ der Projektmitarbeiter_innen stellten.

\section{Interne bzw. externe F\&E-Rekrutierung zur Bearbei- tung des Projekts in $\mathbf{4 9} \%$ der Fälle}

Beschäftigungseffekte bei F\&E-Beschäftigten während bzw. nach der Projektlaufzeit wurden bei 190 der rückmeldenden 390 Projektteilnahmen festgestellt (49\%). In 92 Projektbeteiligungen kam es zu externen Rekrutierungen während der Projektlaufzeit, in 82 zu internen Personalneuzuteilungen. Nach Projektabschluss wurden bei 92 Projektbeteiligungen weitere externe Personen eingestellt, in 42 Beteiligungen wurden bestehende Mitarbeiter_innen intern dem Projekt zugeteilt. Insgesamt wurden 434 Personen für mit den geförderten F\&E-Projekten verbundenen Tätigkeiten extern rekrutiert, 365 intern umgeschichtet. Diese Beschäftigungseffekte gehen großteils auf das Konto von großen Unternehmen, allerdings mit einem rückläufigen Trend, während Kleinunternehmen eine zunehmend wichtigere Rolle spielen.

Bei den Instrumenten Einzelprojekt und Kooperationsprojekt wurde sowohl während dem Projekt, als auch danach, häufiger externes F\&E-Personal rekrutiert als intern umgeschichtet. Auffällig ist, dass bei Kooperationsprojekten mehr externe Mitarbeiter_innen nach als während der Projektlaufzeit eingestellt wurden, d.h. dass in diesen Projekten vermehrt Weiterentwicklungsbedarf bestand.

Hohe technische Zielerreichung - gleichzeitig benötigen viele Projekte noch weitere Entwicklungsschritte

Ein Projekterfolg aus technischer Sicht in dem Sinne, dass zumindest Projektteile nutzenstiftende Ergebnisse lieferten, konnte in $94 \%$ der Projektbeteiligungen erzielt werden (2017: $92 \%)$. Aus wirtschaftlicher Sicht beurteilen fast $65 \%$ das Projekt dahingehend, dass die Projektziele bislang erreicht wurden - damit wurde der 2014 berichtete Höchstwert von 66 \% beinahe erreicht. 
Dieser Wert hängt stark mit den ursprünglichen Erwartungen an das Projekt zusammen - dies macht es umso erstaunlicher, wie konstant auch dieser Wert über die Jahre ist.

Falls die wirtschaftlichen Projektziele nicht erreicht werden konnten, so lag dies in $51 \%$ der Fälle an weiterführenden Entwicklungsarbeiten, die zur Verwertung erforderlich sind (KU: $57 \%$, MU: $39 \%$, GU: $48 \%)$ ). Diese Zunahme ist gänzlich auf Projekte in den Kooperationsformaten zurückzuführen. Seit 2015 ist dieser Wert ausgehend von $40 \%$ kontinuierlich angestiegen, was impliziert, dass die Projekte in leicht anwendungsferneren Entwicklungsstufen realisiert werden. Diese Hypothese steht in Einklang damit, dass 2018 deutlich mehr Befragte angaben, die Fragestellungen in F\&E-Folgeprojekten fortzusetzen.

Ein weiterer wesentlicher Grund zum Nichterreichen der wirtschaftlichen Ziele liegt in den Herstellungskosten, die höher als erwartet ausfielen ( $26 \%$ gegenüber $17 \%$ in den Vorjahren). Dass sich dies negativ auf das Erreichen der wirtschaftlichen Ziele auswirkt, hängt aber nicht unbedingt mit Problemen in der Finanzierung bis zur Marktreife zusammen, die nur für $9 \%$ ein Problem darstellt (2017: $12 \%)$. Schließlich wurde in $27 \%$ der Projektbeteiligungen die Entwicklung der Nachfrage oder des Marktpreises falsch eingeschätzt, was v.a. auf mittlere Unternehmen zutrifft (33\%).

Differenziert nach Unternehmensgröße zeigt sich, dass Großunternehmen höhere wirtschaftliche Erfolgsraten angeben: während $70 \%$ der GU die wirtschaftlichen Projektziele erreichen konnten, ist dies nur bei $60 \%$ der KU bzw. MU der Fall. 2017 lagen GU und KU noch gleichauf bei rd. $64 \%$, MU abgeschlagen bei nur $52 \%$.

\section{3 | Projektergebnisse und deren wirtschaftliche Verwertung}

\section{Wenig Veränderung bei der Art von Innovationen}

In 356 Projektbeteiligungen entstanden 633 innovative (Teil-)Lösungen, d.h. ein Unternehmen konnte im Durchschnitt 1,8 Innovationen aus der jeweiligen Projektbeteiligung entwickeln. Die Arten der Innovationen verteilt sich über die Jahre sehr konstant: der Anteil von neuen Produkten hat sich wieder leicht auf $29 \%$, jener von veränderten Produkten auf $17 \%$ erhöht, während der Anteil an neuen Dienstleistungen nach dem letztjährigen Spitzenwert von $12 \%$ auf $9 \%$ zurückging.
Tabelle 4 Arten der Innovation

\begin{tabular}{lcc}
\hline & \% aller Nennungen & \% der Projekte \\
\hline Neues Produkt & $29 \%$ & $51 \%$ \\
Verändertes Produkt & $17 \%$ & $30 \%$ \\
\hline Neues Verfahren & $16 \%$ & $29 \%$ \\
\hline Verändertes Verfahren & $12 \%$ & $21 \%$ \\
\hline Neue DL & $9 \%$ & $15 \%$ \\
\hline Neues Design & $8 \%$ & $14 \%$ \\
\hline Veränderte DL & $7 \%$ & $13 \%$ \\
\hline Organisation & $3 \%$ & $5 \%$ \\
\hline Nennungen/Projekte & 633 & 356 \\
\hline
\end{tabular}

Quelle: KMU Forschung Austria. Mehrfachnennungen möglich. DL...Dienstleistung. $\mathrm{N}=356$

Absolut gesehen entstanden auch 2018 die meisten Innovationen in Einzelprojekten (326), gefolgt von Kooperationsprojekten (213). In beiden Projektformen werden primär neue Produkte entwickelt, allerdings ist der Anteil von Produktneuheiten bei Einzelprojekten deutlich höher als bei Kooperationsprojekten ( $36 \%$ vs. $22 \%$ ). Stattdessen resultieren Kooperationsprojekte etwa doppelt so häufig in neue oder veränderte Dienstleistungen, während die anderen Innovationsarten in ähnlichem Ausmaß vertreten sind.

Abbildung 5 Arten von Innovationen

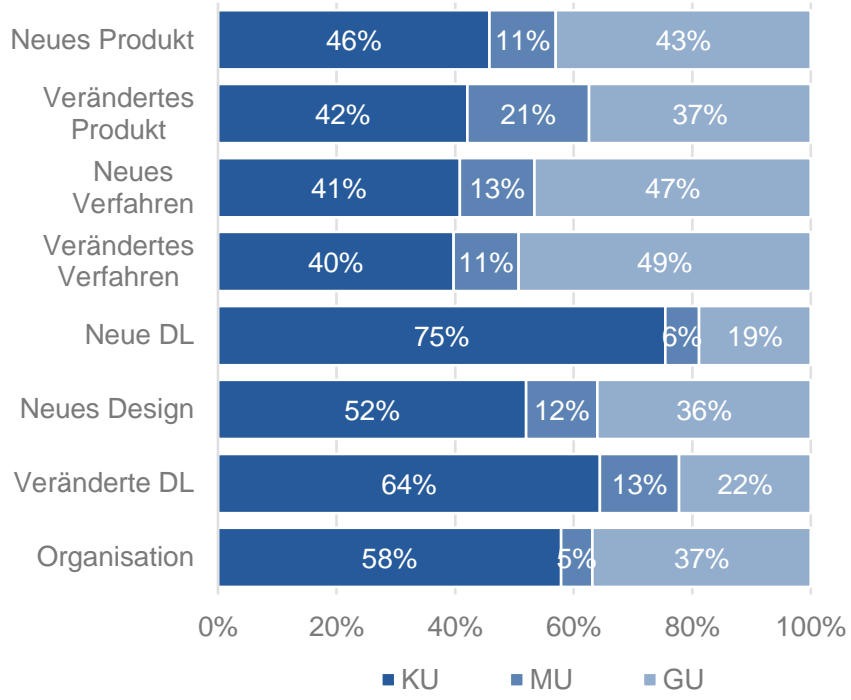

Quelle: KMU Forschung Austria. Hauptergebnisse der Forschungsprojekte. Mehrfachnennungen. DL...Dienstleistung. N=356

Abbildung 5 zeigt die Verteilung der Innovationen aus den im Jahr 2014 abgeschlossenen Projekten nach Unternehmensgröße. Die Anteile bei den Produktneuheiten entsprechen der Verteilung der Unternehmen über die drei Größenklassen, während veränderte Produkte als Innovationsoutput überproportional häufig bei MU entstehen. Neue wie veränderte Dienstleistungen, sowie Design und Organisation werden besonders häufig von $\mathrm{KU}$ entwickelt. 
Schließlich wurde in der Befragung 2018 erstmals erhoben, inwiefern das geförderte F\&E-Projekt einen Einfluss auf ein potenziell neues Geschäftsmodell hatte. Aggregiert war dies in rd. $6 \%$ der Fall, vor allem bei mittleren Unternehmen (11\%), während dies bei kleinen sowie großen Unternehmen kaum zutraf (4\%).

\section{Komplexere Innovationen nehmen zu}

Bei KMU ist im WiMon 2018 wieder ein leichter Anstieg bei reinen Produkt-, sowie reinen Prozessinnovationen zu beobachten. Dies ändert aber nichts an der mittelfristigen Entwicklung, dass kombinierte und damit komplexere Innovationen zunehmen. Dies ist speziell bei der Kombination Produkt mit Dienstleistungen zu beobachten.

Abbildung 6 Innovationen und deren Kombinationen, 2007-2014
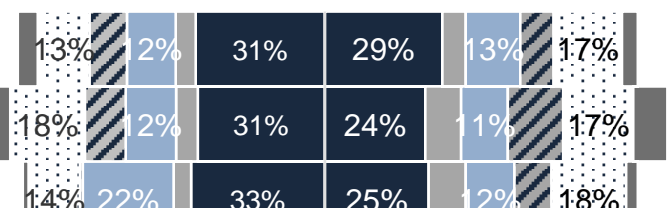

GU

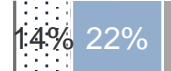

$33 \%$
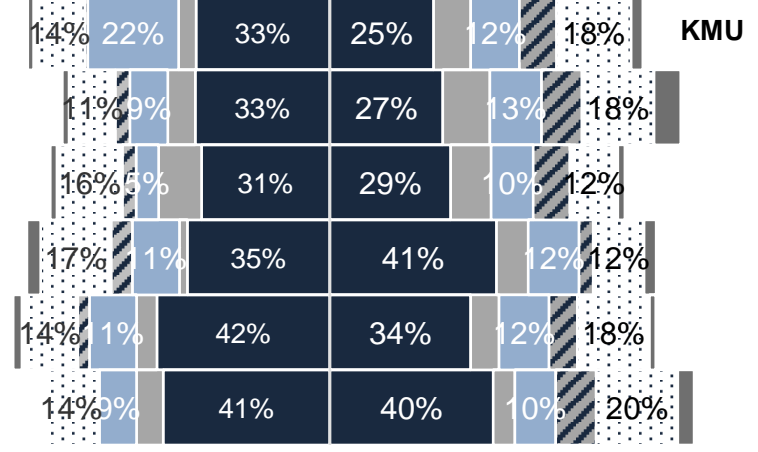

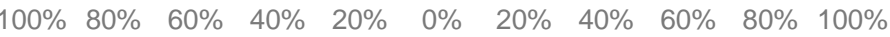

- Nur neues Produkt

- Nur neues Verfahren

- Neues Produkt \& neues Verfahren $\quad$ Neues Verfahren \& neue DL

Quelle: KMU Forschung Austria. Angaben in Prozent aller Projektbeteiligungen mit Innovationen. Mehrfachnennungen möglich.

\section{Gut ein Viertel der geförderten Projekte führte zur Anmeldung von Schutzrechten}

Ähnlich wie in den Vorjahren ergaben sich Schutzrechtanmeldungen in $28 \%$ der Projektbeteiligungen von rückmeldenden Unternehmen (95 Fälle). Über alle GröBenklassen hinweg meldeten bei den im Jahr 2014 abgeschlossenen Projekten mehr Unternehmen Schutzrechte an, am häufigsten aber Großunternehmen. Es bleibt abzuwarten, ob der neu eingeführte Patentscheck der FFG hier in den nächsten Jahren Änderungen hervorrufen wird.
Abbildung 7 Anmeldung von Schutzrechten in Folge des geförderten F\&E-Projekts, Projektende 2008-2014

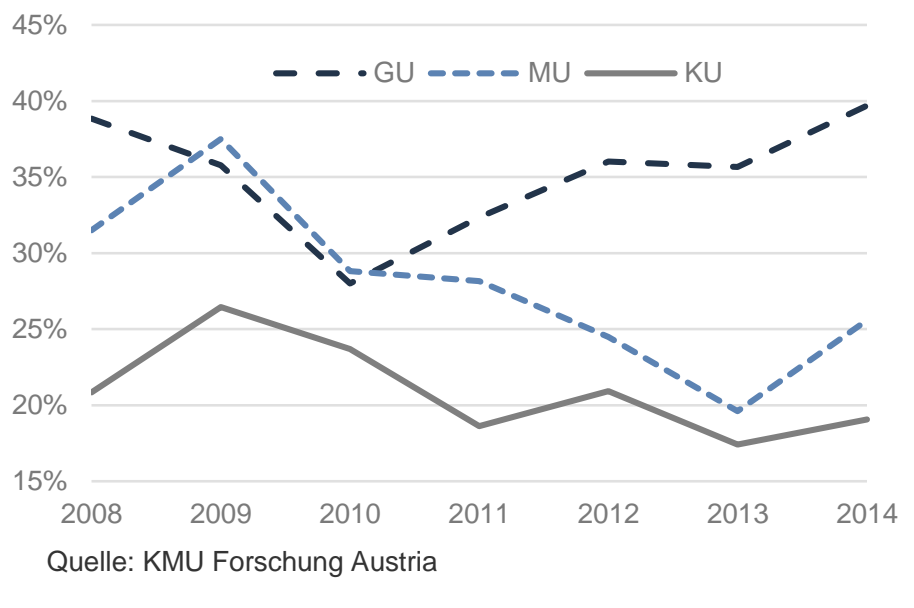

Insgesamt meldeten die Unternehmen 396 Patente alleine oder mit Projektpartnern an - diese hohe Zahl (2017: 254) wird von zwei Projekten dominiert, in welchen High-Tech Start-Ups insgesamt 152 Patente anmeldeten. Ohne diese zwei Projekte entspricht die Anzahl der technischen wie nichttechnischen Schutzrechte jener der Vorjahre: 2,8 technische Schutzrechte pro Unternehmen und 1,5 nicht-technische. Einzig bei den Gebrauchsmustern ist eine deutliche Steigerung zu beobachten.

\section{Weniger Neuheiten am internationalen Markt}

Auf Ebene der Projektbeteiligung schätzen die Unternehmen $64 \%$ der aus den Projekten resultierenden Innovationen als Neuheiten auf ihrem internationalen Markt ein. In rund $14 \%$ der Projektbeteiligungen erwiesen sich die Innovationen als Neuheit auf dem österreichischen Markt, in $22 \%$ der Fälle stellen sie eine Unternehmensneuheit dar. Der hohe Anteil an Unternehmensneuheiten geht auf Großunternehmen wie auch mittlere Unternehmen zurück und hängt einerseits mit neu entwickelten Verfahren und andererseits mit verbesserten Produkten zusammen.

Wie auch in den vergangenen Jahren zeigt sich nach Instrumenten, dass internationale Neuheiten vorrangig aus Einzelprojekten entstehen (73\%, 2017: $75 \%$ ), gefolgt von Kooperationsprojekten (58\%, 2017: $64 \%$ ).

\section{Verbesserung der technologischen Wettbewerbs- position}

Auf nationaler Ebene befinden rund $35 \%$ der Unternehmen, dass die Projektteilnahme dazu beigetragen hat, die Position an der technologischen Spitze zu halten bzw. zu dieser aufzuschließen (2017: $32 \%$ ), auf internationaler Ebene konstatieren dies 33\% (2017: $29 \%$ ). 
Wie auch in den Vorjahren trifft dies besonders häufig auf Unternehmen im Basisprogramm zu (44\% national wie international), in den übrigen Programmen ist dieser Anteil niedriger (26\% national, $24 \%$ international).

Wenn Unternehmen das Projektthema weiterverfolgten und dafür auch eine Förderung in Anspruch nahmen, so ist die Verbesserung der Wettbewerbsposition deutlich stärker: in $48 \%$ (44 \%) konnte auf nationaler (internationaler) Ebene zur Spitze aufgeschlossen werden bzw. die Spitzenposition gehalten werden. Dies entspricht auch den Werten, wenn das Unternehmen eine weitere FFG-Förderung erhalten hat.

Die Hälfte der Projektergebnisse werden vier Jahre nach Projektende bereits verwertet - aber die Verwertung verschiebt sich weiter in die Zukunft

Tabelle 5 Wirtschaftliche Verwertung der vor vier Jahren abgeschlossenen Projekte, 2018

\begin{tabular}{lcccc}
\hline & $\mathbf{n}$ & In \% & $\begin{array}{c}\text { Barwert } \\
\text { (Mio. } € \text { ) }\end{array}$ & In \% \\
\hline Bereits wirt. verwertet & 186 & $50 \%$ & 38,5 & $53 \%$ \\
\hline In Zukunft wirt. verwertet & 67 & $18 \%$ & 18,3 & $25 \%$ \\
$\begin{array}{l}\text { Keine wirt. Verwertung, aber } \\
\text { Erkenntnisgewinn }\end{array}$ & 82 & $22 \%$ & 10,4 & $14 \%$ \\
Kann nicht verwertet werden & 13 & $3,5 \%$ & 2,4 & $3 \%$ \\
\hline $\begin{array}{l}\text { Kein unmittelbares Verwer- } \\
\text { tungsziel }\end{array}$ & 24 & $6,5 \%$ & 3,1 & $4 \%$ \\
\hline Gesamt & 372 & $100 \%$ & 72,7 & $100 \%$ \\
\hline
\end{tabular}

Quelle: KMU Forschung Austria. N=372

In der Erhebung 2018 setzt sich die Entwicklung fort, dass ein geringerer Anteil des Barwerts des gesamten Fördervolumens in Projekte fließt, die bereits wirtschaftlich verwerten werden. Im Vorjahr betrug der Anteil $55 \%$; der Durchschnitt im Zeitraum 2011-2016 lag hingegen bei $67 \%$. Stattdessen fließen größere Anteile in Projekte, deren Ergebnisse erst in Zukunft verwertet werden können (2017: 20 \%, 2011-2016: $12 \%$ ).

Der hohe Wert geht bei im Jahr 2014 beendeten Projekten damit einher, dass in überdurchschnittlich vielen Projekten noch weitere Entwicklungsschritte zur Verwertung notwendig sind.
Abbildung 8 Wirtschaftliche Verwertung, Projektende 2011-2014

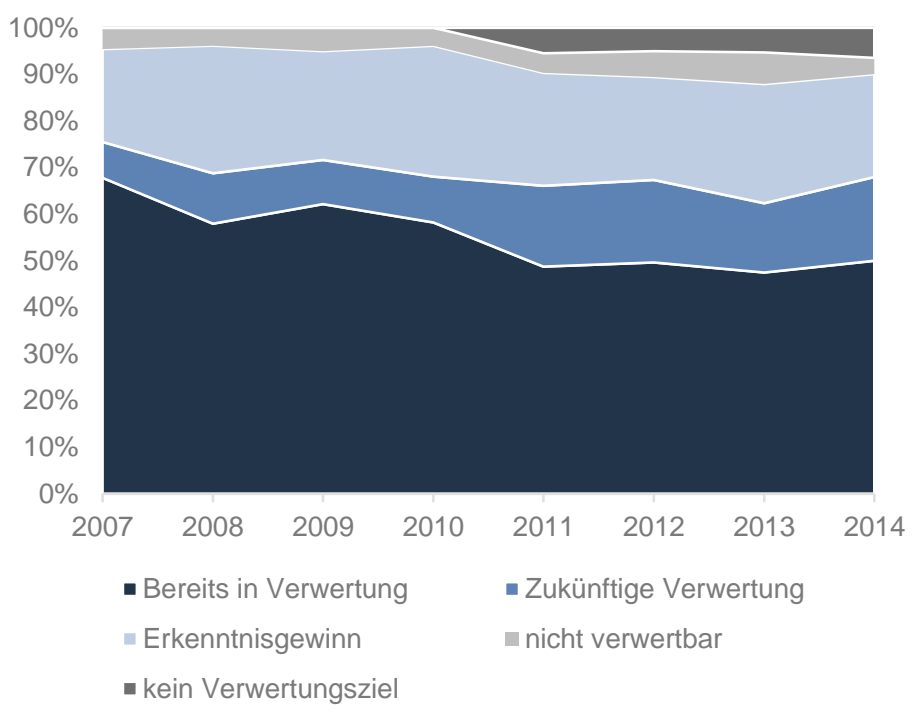

Quelle: KMU Forschung Austria

Insgesamt zeigt sich wie auch in den vergangenen Jahren, dass GU schneller verwerten als KU oder MU und dass produzierende Unternehmen (über alle Technologiegruppen hinweg) tendenziell schneller verwerten als Unternehmen im Bereich der (wenig) wissensintensiven Dienstleistungen.

$65 \%$ der Einzelprojekte werden innerhalb von vier Jahren wirtschaftlich verwertet; Projekte in anderen Instrumenten benötigen mehr Zeit

Differenziert nach den Instrumenten Einzel- und Kooperationsprojekt zeigen sich deutliche Unterschiede, welche die dahinterliegenden Interventionslogiken widerspiegeln. Während in Einzelprojekten bereits $65 \%$ der Ergebnisse wirtschaftlich verwertet werden, sind es bei Kooperationsprojekten erst $32 \%$; in 17\% der Kooperationsprojekte ist die Verwertung in der Zukunft geplant, in $35 \%$ dient das F\&E-Projekt dem Erkenntnisgewinn.

Von jenen Projekten mit einem wirtschaftlichen Verwertungsziel liegen zu 228 Projektbeteiligungen Informationen über den Zeitpunkt der wirtschaftlichen Verwertung bzw. Einschätzungen vor, ab welchem Jahr eine Verwertung möglich war bzw. sein wird. In $77 \%$ der Fälle wurden die Ergebnisse innerhalb von vier Jahren nach Projektende verwertet, $23 \%$ beanspruchen einen längeren Zeithorizont. 
Abbildung 9 Zeitpunkt der wirtschaftlichen Verwertung, Einzel- und Kooperationsprojekte

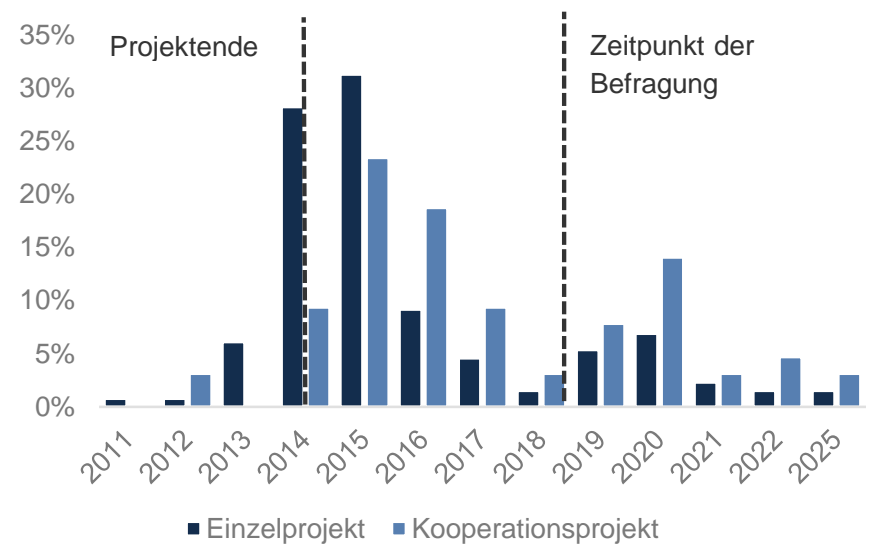

Quelle: KMU Forschung Austria, $\mathrm{n}=228$

\section{Projektverwertung konnte Arbeitsplätze sichern}

Bei 147 Unternehmen (58\% der Projektteilnahmen mit wirtschaftlichem Verwertungsziel) resultierte aus der wirtschaftlichen Verwertung der Projektergebnisse ein direkter Beschäftigungseffekt von 1.878 Arbeitsplätzen (1.392 gesicherte und 489 neu geschaffene Arbeitsplätze, nach Köpfen).

Im Vergleich zum Vorjahr ist die Beschäftigungswirkung zwar insgesamt ähnlich hoch, jedoch entfielen bei 2013 abgeschlossenen Projekten $54 \%$ auf neu geschaffene Arbeitsplätze, während es bei Projektbeteiligungen mit Projektende 2014 lediglich $26 \%$ sind. Dies stellt auch den mit Abstand niedrigsten Wert im WiMon dar und reflektiert die Stagnation der österreichischen Wirtschaft zwischen 2012 und 2015. In Kleinunternehmen entfallen dennoch $65 \%$ der Beschäftigungswirkung auf neu geschaffene Arbeitsplätze, bei mittleren Unternehmen sind noch $43 \%$, bei Großunternehmen aber nur $13 \%$.

\section{Wirtschaftliche Verwertung vorranging auf auslän- dischen Märkten}

Gesicherte Umsätze, zusätzliche Umsätze sowie Lizenzerlöse resultieren primär aus der Verwertung der Projektergebnisse auf ausländischen Märkten. Wohl aufgrund der mäßigen Entwicklung der österreichischen Wirtschaft im Beobachtungszeitraum liegen die im Export erwirtschafteten Anteile an allen drei Einnahmequellen bei Projekten mit Projektende 2014 noch höher als in den vergangenen Jahren ( $r d .93 \%$ ).
Abbildung 10 Anteil der gesicherten bzw. zusätzlichen Umsätze bzw. Exporte, die im Ausland erwirtschaftet wurden. Projektende 2008-2014

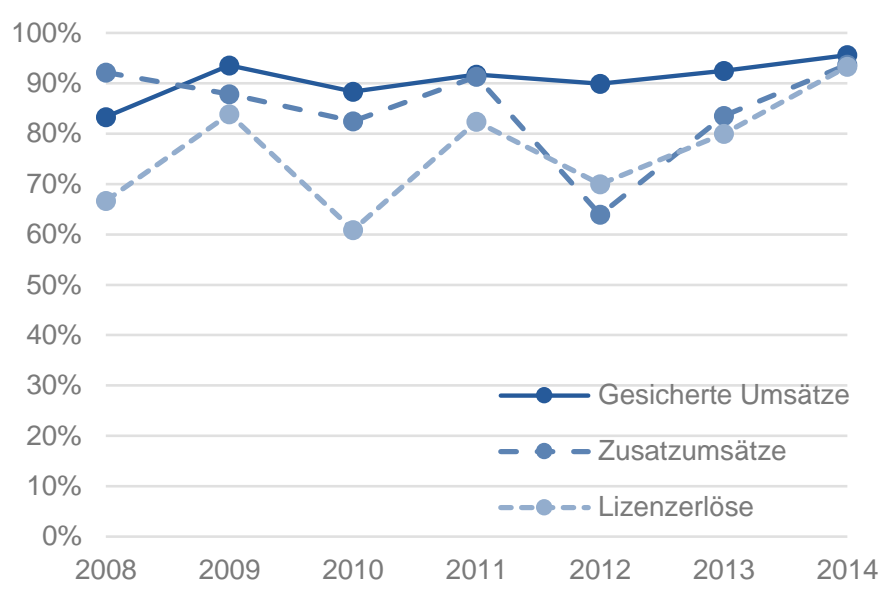

Quelle: KMU Forschung Austria

Der Anteil an Unternehmen mit Lizenzerlösen liegt bei der Befragung 2018 bei $10 \%$ (2017: $13 \%, 2016: 11 \%$ ), jener mit Zusatzerlösen bei $65 \%$, was dem langjährigen Mittel entspricht. Allerdings berichten nur $47 \%$ der Unternehmen, dass durch die Verwertung Umsätze gesichert wurden. Dies stellt zwar doch einen recht deutlichen Rückgang zu den zwei Jahren zuvor dar, liegt aber im Schwankungsbereich 2011-2018.

Von den 18 Unternehmen, die Lizenzerlöse vermelden, sind 14 Kleinunternehmen im Basisprogramm.

Bei 42 Unternehmen ist bislang keine Lizenzierung der Projektergebnisse erfolgt, obwohl das Projektergebnis prinzipiell lizenzierbar ist. Insgesamt erwirtschafteten die Unternehmen mit der Lizenzvergabe bisher rund $€$ 7,7 Mio. (2017: € 15 Mio., 2016: € 7,7 Mio.).

Zusätzliche Umsätze konnten im Zuge von insgesamt 71 Projektteilnahmen erzielt werden $(65 \%$ der Unternehmen, die die Projektergebnisse zum Zeitpunkt der Befragung bereits wirtschaftlich verwerten) -51 Unternehmen machten eine Auskunft zur Höhe der Zusatzumsätze. Insgesamt berichten sie von $€ 411$ Mio. - ein Rückgang im Vergleich zu 2017 ( $€ 613$ Mio.), allerdings ähnlich hoch wie im Jahr 2016 ( $€ 490$ Mio.)

\section{Geringerer Fördermultiplikator aufgrund der kon- junkturellen Entwicklung}

Der Fördermultiplikator stellt eine simple Input-/OutputRelation der geförderten Projekte dar. Als Input wird der Barwert der vergebenen Fördermittel herangezogen, jenes für den Output ist die Summe der Lizenzerlöse und Zusatzumsätze (durch die Verwertung der Projekter- 
gebnisse). Dieser wird für jedes Unternehmen berechnet und dann aggregiert. Aufgrund der Einfachheit dieses Indikators ist weniger die absolute Höhe interessant, sondern die Unterschiede zwischen Instrumenten sowie die Veränderung im Zeitverlauf.

Abbildung 11 zeigt die Höhe des Multiplikators der FFGFörderungen für das Basisprogramm und die übrigen Programme. Wie auch in den vergangenen Jahren ist der Fördermultiplikator des Basisprogramms deutlich höher als jener der übrigen Programme.

Abbildung 11 Fördermultiplikator für das Basisprogramm und die übrigen Programme, Projektende 2007-2014

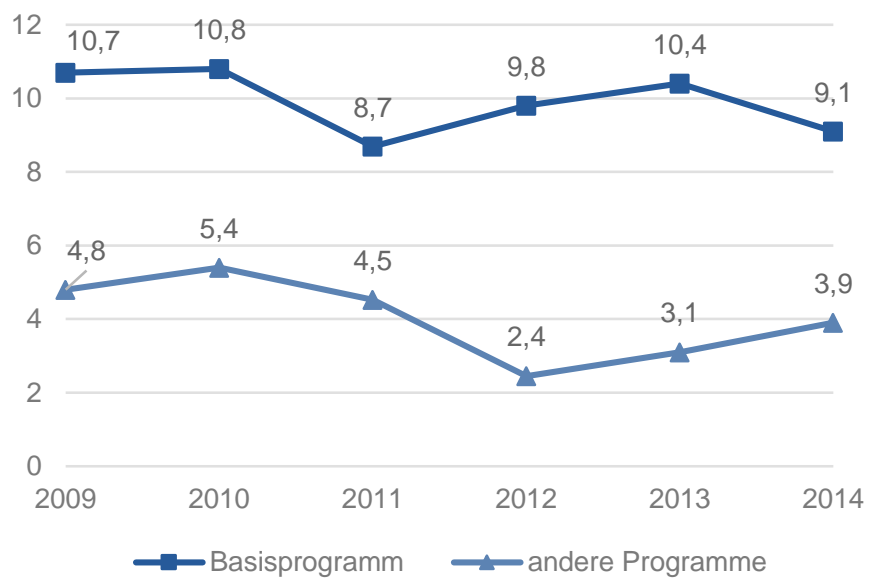

Quelle: KMU Forschung Austria

\section{4 | Additionalität}

\section{4 \% tätigen Zusatzinvestitionen, um die Projekter-} gebnisse auf dem Markt zu platzieren

Im WiMon 2018 wurden erstmals Informationen erhoben, inwiefern zusätzliche Investitionen bzw. Ausgaben notwendig waren, um die Projektergebnisse am Markt zu platzieren. $64 \%$ der Unternehmen, die mit dem Projekt auf eine wirtschaftliche Verwertung abzielen, geben an, weitere Investitionen getätigt zu haben. Der Anteil nimmt jedoch mit der Unternehmensgröße ab: $72 \%$ der KU tätigten weitere Ausgaben, $61 \%$ der MU und $57 \%$ der GU. Hinsichtlich der Instrumente treten keine Unterschiede auf.

102 Unternehmen machten Angaben zur Höhe der Investitionen: insgesamt wurde ein Volumen $€ 67,5$ Mio. investiert. $67 \%$ davon entfallen auf GU, $27 \%$ auf $\mathrm{KU}$ und lediglich $6 \%$ auf MU. Im Median tätigte ein KU zusätzliche Investitionen in Höhe von $€ 110.000$, ein MU von $€ 200.000$ und ein GU von $€ 500.000$.
Setzt man die zusätzlichen Investitionen in Bezug zum Barwert der Förderung, so kann eine direkte Wirkung der Förderung auf der Input-Ebene angenähert werden. In $65 \%$ der Projektbeteiligungen investierten die Unternehmen mehr, als sie als Förderung für das jeweilige Projekt erhielten. Großunternehmen und Kleinunternehmen investierten im Median beinahe das Doppelte der Fördersumme, um die Projektergebnisse auf dem Markt zu platzieren, bei mittleren Unternehmen sind es rd. $120 \%$. Dies kann als Anzeichen für eine positive Input-Wirkung gewertet werden, auch wenn diese einfache Analyse keine Aussagen über die tatsächliche Additionalität der Förderung erlaubt.

\section{$€ 58$ Mio. Investitionen in F\&E-Infrastruktur im Zu- sammenhang mit den Projekten}

Ähnlich wie in den vergangenen Jahren investierten rund $40 \%$ der Unternehmen im Rahmen des geförderten F\&E-Projekts in die F\&E-Infrastruktur. Besonders häufig ist dies bei Unternehmen im Instrument Einzelprojekt der Fall (51\%), während nur ein Viertel der Unternehmen mit Kooperationsprojekten solche Investitionen tätigten.

125 Unternehmen quantifizierten die Höhe der Investitionen - diese summieren sich auf rd. $€ 58 \mathrm{Mio}$. Im Median investierte ein Unternehmen rd. $€ 0,5$ je Fördereuro, im Durchschnitt sind es jedoch $€ 5,2$. Dies verdeutlicht, wie ungleich sich die Investitionen über die Unternehmen verteilen. Setzt man die F\&E-Infrastrukturinvestitionen in Relation zur gesamten Fördersumme, die an die Unternehmen im WiMon ausbezahlt wurde, so wurden im Durchschnitt $€ 0,75$ je Fördereuro investiert.

Die Gesamtsumme der Investitionen in F\&E-Infrastruktur wird von relativ wenigen Projektteilnahmen geprägt. Diese können aber nicht ausschließlich dem geförderten Projekt zugeschrieben werden, sondern sind eher Bestandteil umfangreicher Entwicklungsarbeiten und der strategischen Ausrichtung des Unternehmens.

\section{Steigende Additionalität in konjunkturell schwieri- ger Lage}

$39 \%$ der befragten Unternehmen berichten, dass sie das Forschungsprojekt ohne die FFG-Förderung nicht durchgeführt hätten, in $51 \%$ der Fälle wäre das Projekt in geringerem Ausmaß durchgeführt worden. Bei rd. $9 \%$ der Projektteilnahmen trat ein (gewisses) Crowding-out privater Innovationsausgaben ein, als in diesen Fällen das Forschungsprojekt entweder vollständig $(2,6 \%)$ oder in überwiegendem Ausmaß $(6,8 \%)$ auch 
ohne Förderung vorangetrieben worden wären. Dieser Effekt liegt bei marktnäheren Projekten der Experimentellen Entwicklung naheliegender Weise höher.

Im internationalen Vergleich liegt dieser Wert eher am unteren Rand. So führen in Deutschland z.B. rund $15 \%$ der Unternehmen, die um eine Förderung im Rahmen des Zentralen Innovationsprogramms Mittelstand ansuchen, das Projekt auch ohne Fördergelder durch.

Insgesamt zeigt sich im Monitoring der Projekte, die im Jahr 2014 abgeschlossen wurden, die bisher größte Additionalität. Im Kontext des schleppenden Konjunkturverlaufs während der Projektantragsphase ist die starke Wirkung auf der Förderung auf die Projektdurchführung plausibel und zeigt auch das antizyklische Potenzial der F\&E-Förderung.

Abbildung 12 Additionalität der F\&E-Förderungen. Hätten Sie das Projekt auch ohne Förderung durchgeführt? Projektende 2008-2014

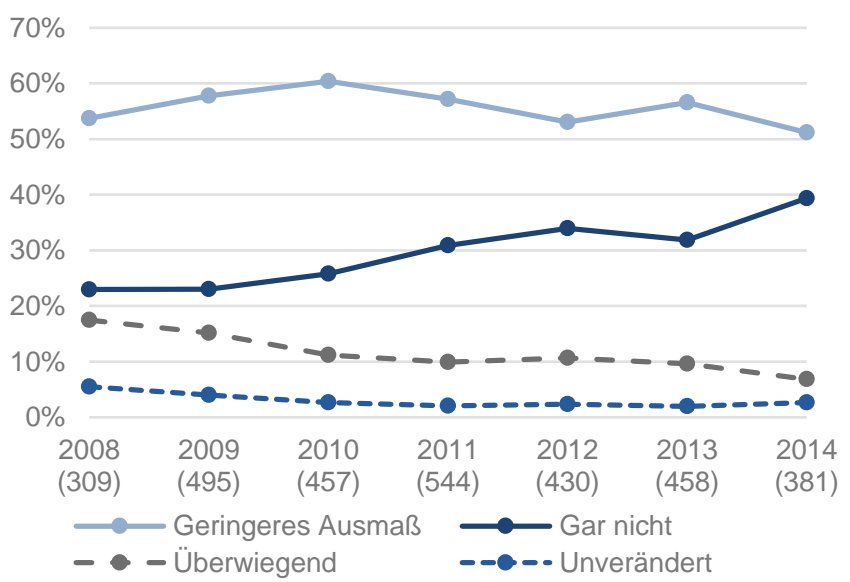

Quelle: KMU Forschung Austria. Jahr der Beendigung des F\&E-Projekts, Anzahl an Fällen in Klammern.

Zu zeitlichen Verzögerungen wäre es - wie auch im Vorjahr - in rd. $90 \%$ aller Vorhaben gekommen. Mögliche Auswirkungen auf die Kooperation mit Partnern haben $45 \%$ der Befragten angenommen, d.h. das Projekt wäre mit weniger Kooperationspartnern durchgeführt worden. Der Rückgang im Vergleich zum Vorjahr (54\%) resultiert daraus, dass weniger Kooperationen geplant waren (31\%, 2017: $27 \%)$ sowie dass die Förderung keinen Einfluss auf das Kooperationsverhalten hatte (25\%, 2017: $19 \%)$.

Auch 2018 zeigt sich wieder, dass die Bereitschaft bzw. die Fähigkeit, Projekte ohne Fördermittel durchzuführen mit der Unternehmensgröße steigt (keine Projektumsetzung ohne Förderung: KU $48 \%$, MU $38 \%$, GU $30 \%$ ). Nach Technologiefeldern wären am stärksten Unter- nehmen im Bereich der wissensintensiven Dienstleistungen $(47 \%)$ sowie der weniger wissensintensiven Dienstleistungen (40\%) betroffen.

Besonders häufig würden Kooperationsprojekte ohne die Förderung nicht umgesetzt werden - dies befinden $55 \%$ der Unternehmen, während das nur auf $22 \%$ der Unternehmen mit Einzelprojekten zutrifft. Bei den Kooperationsprojekten erhöhte sich die Additionalität im Vergleich zum Vorjahr um $12 \%$-Punkte, bei Einzelprojekten um 3 \%-Punkte. 


\section{4 | Wirkungen in Forschungseinrichtungen}

\section{1 | Rolle und Positionierung der F\&E-Projekte}

\section{Rolle von Forschungseinrichtungen als Impulsgeber für F\&E-Projekte nimmt $a b$}

Der Impuls zur Durchführung der Projekte ist in knapp $37 \%$ der Fälle von den FE ausgegangen. Seltener haben Fachhochschulen (17\%) ihre Ideen an Unternehmen herantragen, während dies bei AUF deutlich öfter erfolgte $(43 \%)$.

Der Anteil an Projektideen, die von der Forschungseinrichtung stammen, ist seit 2015 von rd. $46 \%$ kontinuierlich gesunken. Stattdessen werden häufiger Projektideen mit den Unternehmenspartnern gemeinsam entwickelt (2015: $28 \%$, 2018: $33 \%$ ) bzw. stammen direkt von einem Unternehmen (2015: $23 \%$, 2018: $26 \%$ ), was auf eine zunehmende Vernetzung der Forschungspartner deutet.

\section{Der hohe Anteil an Vorprojekten zeigt die kumula- tive Natur der Forschung}

Im Jahr 2018 berichten 60 \% der Forschungseinrichtungen, dass das FFG-Projekt auf eines bzw. mehrere inhaltlich relevante Vorprojekte zurückzuführen ist (2017: $63 \%)$. Ob Vorprojekte umgesetzt wurden, variiert zwischen den verschiedenen Arten von FE und über die Erhebungsjahre, allerdings nicht sehr systematisch. In den Erhebungen 2014-2017 führten AUF, Fachhochschulen und Kompetenzzentren tendenziell häufiger mehrere Vorprojekte zum gleichen Thema durch als Universitäten - im Jahr 2018 hingegen bauen fast zwei Drittel der Projekte von Universitäten auf mindestens einem Vorprojekt auf. Gleichzeitig impliziert der Anteil an Projekten (33\%, 2017: $29 \%$ ), der keinem Vorprojekt zugeordnet werden kann, dass ein Teil der FE relativ heterogene Projekte umsetzt. Dies steht auch im Einklang damit, dass etwas weniger Projektideen von den FE selbst stammen als 2017 (37\% vs. $40 \%$ 2017).

\section{FFG weiterhin häufigste öffentliche Finanzierungs- quelle von Vorprojekten}

Wie auch in den Vorjahren wurden über $80 \%$ der Vorprojekte öffentlich gefördert - der wichtigste Fördergeber ist erneut die Forschungsförderungsgesellschaft. 70 von $95 \mathrm{FE}$ mit öffentlich mitfinanzierten Vorprojekten (74 \%) wurden von der FFG unterstützt. $15 \%$ der FE erhielten Mittel der Europäischen Kommission, $11 \%$ vom FWF - in beiden Fällen häufig in Kombination mit anderen Förderungen. Die übrigen Finanzierungsquellen sind andere Institutionen (z.B. auf Länderebene).

Der Anteil an Forschungseinrichtungen, deren Vorprojekte zu einem bestimmten Ausmaß durch Eigenfinanzierung der Institute umgesetzt wurden, liegt bei $23 \%$ (2017: $28 \%$ \%). Schließlich wurden $48 \%$ der FE mit relevanten Vorprojekten auch von Unternehmen finanziert (2017: $40 \%$ ).

\section{$60 \%$ der Projektteilnahmen in bestehenden thema- tischen Schwerpunkten}

Der Anteil an Projekten in bestehenden thematischen Schwerpunkte der jeweiligen FE ist nach dem Anstieg der letzten Jahre wieder etwas gesunken und liegt bei $60 \%$. In $32 \%$ der Fälle war das Projekt die Grundlage eines neuen Schwerpunkts, in den übrigen $8 \%$ streifte es einen Randbereich.

Wie auch im Vorjahr setzen Fachhochschulen am häufigsten Projekte um, die zur Grundlage eines neuen thematischen Schwerpunkts werden (50 \%, 2017: $57 \%$ ) zu nennen ist hier insbesondere COIN im Bereich Strukturprogramme. Bei AUF und Universitäten liegen diese Anteile bei $35 \%$ (2017: $24 \%$ ) und $30 \%$ (2017: $27 \%$ ).

Auf Programmebene führten Projekte in den Programmen COIN und Energie der Zukunft zu den meisten neuen thematischen Schwerpunkten. Nach Instrumenten ist im WiMon 2018 der Wissenschaftstransfer jenes, in welchem die meisten neuen Schwerpunkte gesetzt werden $(40 \%)$. Der stärkste Fokus auf bestehende Schwerpunkte ist in Kooperationsprojekten.

Abbildung 13 Primäre Rolle des geförderten Projekts im Portfolio der Forschungseinrichtung; Nach Instrumenten

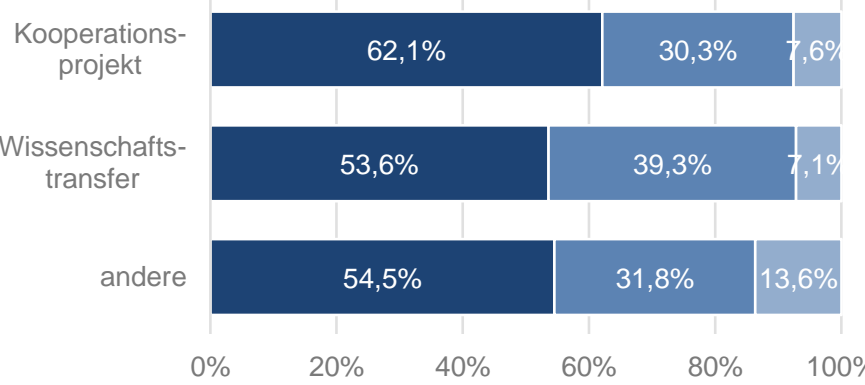

- Bestehnder Schwerpunkt - Neuer Schwerpunkt - Randbereich Quelle: KMU Forschung Austria; N=210; „andere“ ( $n=44)$ umfasst die Instrumente C3 Einzelprojekt, C5 Leitprojekt, C8 Plattform und C9 Strukturaufbau und C10 Innovationsnetzwerk 


\section{Ein Drittel der Projektteilnahmen ist Teil größerer Vorhaben}

Ähnlich wie im Vorjahr stufen $34 \%$ der Forschungseinrichtungen ihre Projektteilnahme als Bestandteil größerer Forschungsvorhaben ein. Umgekehrt werden $66 \%$ der Projektteilnahmen als Einzelvorhaben angesehen insbesondere in den Instrumenten Wissenschaftstransfer und Innovationsnetzwerk. In Kooperationsprojekten und den übrigen Instrumenten sind die geförderten Projekte häufiger in größere Vorhaben bzw. Projektbündel eingebunden.

\section{In $60 \%$ der Projetteilnahmen entstehen Folgepro- jekte, die zu $45 \%$ der Fälle wieder von der FFG un- terstützt werden}

Im WiMon 2018 wurden innerhalb von vier Jahren nach Projektende in $80 \%$ Projektbeteiligungen Folgeprojekte gefördert und durchgeführt (Vorjahre: $57 \%$ bis $63 \%$ ). Daraus entstanden mindestens 282 verschiedene Projekte - im Durchschnitt folgten 2,3 Projekte auf das FFG-Projekt (2017: 2,4). Diese Folgeprojekte werden meist mit Teilen des bisherigen Konsortiums, aber auch mit neuen Partnern umgesetzt.

Die wichtigste Finanzierungsquelle von Nachfolgeprojekten ist wiederum die FFG mit $45 \%$ bzw. 152 Projekten (2014: $49 \%$, 2015-2017: rd. $42 \%) .13 \%$ der Folgeprojekte werden durch Mittel der EU finanziert (20142017: 9-15\%) - von jenen 45 Folgeprojekten sind 36 $(80 \%)$ Projektteilnahmen dem Instrument Kooperationsprojekt zuzuordnen. Direkt durch Unternehmen und Institutionen werden $22 \%$ der Folgeprojekte finanziert (2014-2017: $20 \%$ ), eine zumindest teilweise Eigenmittelfinanzierung erfolgt in $8 \%$ der Folgeprojekte (Vorjahre rd. $9 \%$ ).

\section{FFG-Förderung ermöglicht durchgängige Projekt- ketten}

Bei 122 Projektbeteiligungen entstanden Folgeprojekte, von welchen 107 FE die FFG als Finanzierungsquelle für die insgesamt 152 Folgeprojekte nennen. Bei 78 Forschungseinrichtungen, bei welchen das Projekt in Folgeprojekte mündete, wurden relevante Vorprojekte durch eine öffentliche Einrichtung gefördert. Hierzu gibt es aufgrund von Mehrfachnennungen keine exakten Zahlen, allerdings werden rund $74 \%$ dieser Projekte ebenfalls durch die FFG finanziert. Daraus ergibt sich, dass bei Projekten mit Projektende 2014 die FFG in rund $47 \%$ der Projektbeteiligungen das untersuchte Projekt sowie die Folgeprojekte förderte, und mit einer großen Wahrscheinlichkeit auch etwaige Vorprojekte. Dieser Anteil ist relativ konstant über die Jahre.

\begin{tabular}{|c|c|c|c|}
\hline $\begin{array}{l}\text { Jahr } \\
\text { Projekt- } \\
\text { ende }\end{array}$ & $\begin{array}{l}\text { Anteil Folge- } \\
\text { projekt -Vor- } \\
\text { projekt }\end{array}$ & $\begin{array}{l}\text { Anteil Folgepro- } \\
\text { jekt - Vorprojekt } \\
\text { mit öff. Förderung }\end{array}$ & $\begin{array}{l}\text { Anteil Folgeprojekt } \\
\text { FFG - Vorprojekt } \\
\text { mit öff. Förderung }\end{array}$ \\
\hline 2010 & $62,2 \%$ & $52,7 \%$ & $40,5 \%$ \\
\hline 2011 & $65,1 \%$ & $53,2 \%$ & $46,8 \%$ \\
\hline 2012 & $61,4 \%$ & $51,4 \%$ & $44,3 \%$ \\
\hline 2013 & $69,2 \%$ & $54,9 \%$ & $46,6 \%$ \\
\hline 2014 & $63,9 \%$ & $52,5 \%$ & $46,7 \%$ \\
\hline
\end{tabular}

Quelle: KMU Forschung Austria. Rund $75 \%$ der öffentlich geförderten Vorprojekte wurden durch die FFG finanziert.

Diese Auswertung veranschaulicht, dass zwischen $61 \%$ und $70 \%$ der untersuchten Projekte keine alleinstehenden Projekte sind, sondern auf Vorprojekte aufbauen und in Folgeprojekte münden.

\section{2 | Projektumsetzung}

\section{Weiter hohe Zahl an F\&E-Beschäftigten}

Für die Umsetzung der F\&E-Tätigkeiten wurden in 194 Projektbeteiligungen 1.088 Mitarbeiter_innen zur Bearbeitung der Aufgabenstellungen einbezogen - der Durchschnitt pro Projekt beträgt 5,4. Wie auch in den Vorjahren wurden die an der Beschäftigtenzahl gemessen größten Projekte von Fachhochschulen abgewickelt $(8,1)$, gefolgt von Universitäten, die 5,5 Personen pro Projekt einsetzten.

Tabelle 7 Am Projekt beteiligte F\&E-Mitarbeiter_innen

\begin{tabular}{lccccc}
\hline & & \multicolumn{4}{c}{ Durchschnitt pro Projekt } \\
\hline & $\begin{array}{c}\text { Ge- } \\
\text { samt }\end{array}$ & $\begin{array}{c}\text { Dip- } \\
\text { lom }\end{array}$ & $\begin{array}{c}\text { Pre } \\
\text { Doc }\end{array}$ & $\begin{array}{c}\text { Post } \\
\text { Doc }\end{array}$ & $\begin{array}{c}\text { Sons- } \\
\text { tige }\end{array}$ \\
Universitäten & 515 & 2,7 & 1,7 & 0,8 & 1,4 \\
AUF & 350 & 1,2 & 1,0 & 1,3 & 2,7 \\
FH & 129 & 5,1 & 1,2 & 1,0 & 2,7 \\
Kompetenzzen- & 79 & 1,7 & 1,4 & 1,3 & 1,5 \\
tren & & & & & \\
\hline
\end{tabular}

Quelle: KMU Forschung Austria; $N=187$, ohne Zentren, Cluster, Netzwerke $(n=3)$ sowie kooperative FE $(n=4)$

In jeweils $77 \%$ der rückmeldenden Projektteilnahmen wurden Diplomand_innen und Doktorand_innen, in $61 \%$ Post Docs und in $87 \%$ sonstiges Forschungspersonal eingesetzt (z.B. Labormitarbeiter_innen oder Dozent_innen). 
Gut ein Drittel waren Mitarbeiter_innen auf Ebene der Diplomanden_innen (inkludiert Bachelor- und Masterstudierende), $22 \%$ fallen in die Kategorie Pre Doc. Rund $44 \%$ war Forschungspersonal auf der Ebene von Post Docs, Labormitarbeiter_innen/ Dozent_innen. Somit waren $56 \%$ der Projektmitarbeiter_innen Auszubildende - ein kontinuierlicher Anstieg ausgehend von 47 \% im Jahr 2014.

Die Projekte mit der höchsten Beschäftigtenzahl sind bei den Kooperationsprojekten zu finden - hier wurden 5,1Mitarbeiter_innen eingesetzt (in der Sammelkategorie „andere“ waren es sogar 7,9). Insgesamt sind die Unterschiede über die Instrumente sowie im Zeitverlauf nur gering.

Die Größe der Projektteams lag zwischen einer und 24 Personen, ähnlich wie in den Vorjahren wurden in $67 \%$ der Projekte bis zu fünf Mitarbeiter_innen eingesetzt.

\section{Zielerreichung auf hohem Niveau}

Rund $65 \%$ der FE konnten die eigenen Projektteile in vollem Umfang umsetzen, weitere $31 \%$ in weiten Teilen. Nach Einschätzung der FE wurden in $49 \%$ auch die Ziele des Gesamtprojektes erreicht; bei weiteren $45 \%$ „trifft [dies] ziemlich zu“. Die Zielerreichung auf beiden Ebenen ist somit bei den Projekten, die im Jahr 2014 abgeschlossen wurden, relativ hoch.

Die höchste Projektzielerreichung wird, wie auch im Vorjahr, bei Projekten im Instrument Wissenschaftstransfer berichtet (72 \%, 2017: $67 \%$ ). Im Instrument Kooperationsprojekt werden in $63 \%$ der Fälle die Ziele des Teilprojekts vollständig erreicht, ein Anstieg um rd. $10 \%$-Punkte im Vergleich zum Vorjahr. Ein ähnliches Bild zeigt sich hinsichtlich der Ziele des Gesamtprojekts.

Dass die Gesamtzielerreichung stets relativ deutlich hinter jener der Teilziele der einzelnen am Projekt beteiligten Forschungspartner liegt, deutet einerseits auf gewisse technologische Risiken bei Kooperationsprojekten und die Komplexität aufgrund der tendenziell steigenden Anzahl an Kooperationspartnern pro Projekt hin.
Weitgehend hohe Zufriedenheit mit Kooperationen, v. a. mit Unternehmen

Rund $95 \%$ der Projekte wurden im Rahmen einer Kooperation umgesetzt. Die Zufriedenheit mit den Kooperationen ist im diesjährigen WiMon insgesamt etwas höher als in den vergangenen Jahren.

Wenn vor dem Forschungsprojekt bereits eine Kooperation der Partner stattfand, ist die Zufriedenheit deutlich höher, als bei einer erstmaligen Zusammenarbeit (Zusammenarbeit mit FE: 60 \% vs. $36 \%$ sehr zufrieden; Zusammenarbeit mit Unternehmen: $67 \%$ vs. $43 \%$ ). Wie auch in den Vorjahren ist die Zufriedenheit in der Zusammenarbeit mit Unternehmen höher als mit FE. Dies impliziert, dass die Forschungseinrichtungen von dem Austausch mit Unternehmen einen besonderen Mehrwert ziehen, der höher ist als der rein wissenschaftliche Austausch mit anderen FE.

Im Falle einer nicht zufriedenstellenden Kooperation hatten die FE die Möglichkeit, Gründe dafür anzuführen. Überwiegend wird eine schwierige Kommunikation, Interessenskonflikte zwischen Unternehmen und der FE, sowie ein teils schwieriges wirtschaftliches Umfeld für die Unternehmenspartner genannt.

Nach einer erstmaligen Kooperation im Rahmen eines FFG-Projekts planen Forschungseinrichtungen eine weitere Zusammenarbeit mit den FE-Partnern in $26 \%$ der Fälle, mit den Unternehmenspartnern in $37 \%$ der Fälle. Im Einklang mit der größeren Zufriedenheit ist auch hier die Zustimmung gestiegen.

Bei längerfristigen Zusammenarbeiten liegt dieser Anteil deutlich höher: In $58 \%$ soll die bestehende Kooperation mit FE-Partnern weitergeführt werden, bei Kooperationen mit Unternehmenspartner soll sie in $67 \%$ der Fälle fortgeführt werden.

\section{3 | Wirkungen der Projekte}

\section{In $61 \%$ der Projekte wurden Vereinbarungen zur Verwertung der Projektergebnisse getroffen}

In rd. $61 \%$ der Projekte wurden Vereinbarungen zur Nutzung und Kommerzialisierung der Projektergebnisse zwischen den Projektpartnern getroffen. In den übrigen $40 \%$ war so eine Vereinbarung nicht relevant oder es gab keine. Diese Werte entsprechen den Vorjahren. 
In $36 \%$ der Projekte lagen die Verwertungsrechte vollständig bei einem Projektpartner, zumeist bei den Unternehmen. Eine 50:50 Aufteilung lag in $33 \%$ der Fälle vor.

In $7 \%$ der Projekte kam es zur Anmeldung von Schutzrechten (2017: $11 \%)$. Die FE sicherten sich in 11 Projektteilnahmen mit 28 Schutzrechten ihre Forschungsergebnisse. Nach dem starken Anstieg vom Vorjahr aufgrund weniger Projekte (allen voran ein Laura Bassi Projekt), liegen diese heuer wieder in dem Bereich der Jahre 2014-2016. Die Anmeldung der Schutzrechte erfolgte vorrangig alleine.

\section{Weiterer Entwicklungsschritt nach Projektende in $80 \%$ der Fälle notwendig}

Die Umsetzungs- und Nutzungsmöglichkeiten der Projektergebnisse werden von den Forschungseinrichtungen vier Jahre nach Projektende unterschiedlich eingestuft. Der vorwettbewerbliche Charakter bzw. die Komplexität der Projekte wird dadurch unterstrichen, dass $80 \%$ der Projektergebnisse noch Entwicklungsschritte benötigten, um konkret anwendbar zu werden (2017: $75 \%$ ). Das Ergebnis bei den FE steht im Einklang mit den Aussagen der befragten Unternehmen, die mit $51 \%$ ebenfalls so häufig wie nie zuvor angeben, dass nach Projektende noch weiterführende Entwicklungsarbeiten notwendig waren bzw. sind, um die Projektergebnisse wirtschaftlich zu verwerten.

Abbildung 14 Wie beurteilen Sie die Umsetzungs- und Nutzungsmöglichkeiten der Projektergebnisse?

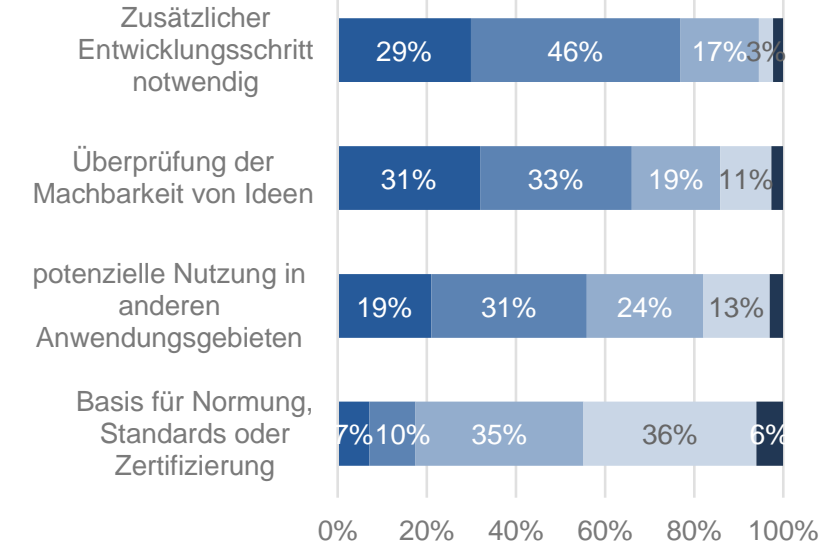

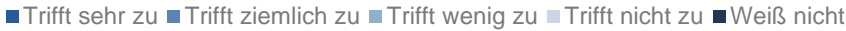

Quelle: KMU Forschung Austria, $n=203$

Im Wirkungsmonitoring 2018 wurde erstmals erhoben, inwiefern die Projektergebnisse als Basis für Normung, Standards oder Zertifizierung dienen können: in $7 \%$ der Fälle trifft dies nach Ansicht der Forscher_innen sehr zu. Aber auch die Frage nach den potenziellen Nutzungen in anderen Anwendungsfeldern deutet auf Möglichkeiten zum Technologietransfer hin, die eine gesonderten Untersuchung bzw. eine erhöhte Aufmerksamkeit seitens der Politik bedürfen könnte.

Neue Kontakte, die Weiterbeschäftigung der wissenschaftlichen Mitarbeiter_innen und der Anstoß von weiterführenden wissenschaftlichen Qualifikationsschriften als wichtigste Wirkungskategorien

In $58 \%$ der Teilnahmen führten die Projekte zu insgesamt 301 weiterführenden Diplomarbeiten oder Dissertationen an den FE (2,6 je Projektteilnahme). Am häufigsten wurden Abschlussarbeiten in Projekten des Wissenschaftstransfers (66\% der Fälle, 2,2 Arbeiten je Projektteilnahme) und den sonstigen Instrumenten (67\%, 4,9 Arbeiten) angestoßen. In $60 \%$ der Fälle wurden nach Projektende insgesamt 220 der beteiligten Diplomand_innen/Dissertand_innen an den FE weiterbeschäftigt, was rund $36 \%$ aller Projektmitarbeiter_innen auf dieser Ausbildungsstufe entspricht.

Abbildung 15 Welche der folgenden Effekte ergaben sich aufgrund des FFG-Projekts? Projektende 2010-2014

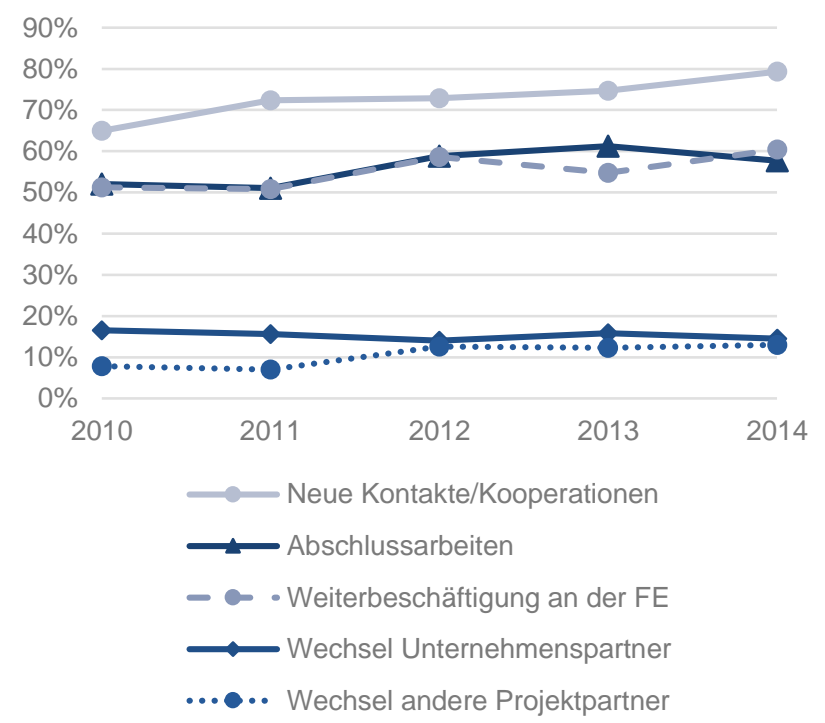

Quelle: KMU Forschung Austria

Der Wechsel von F\&E-Personal zu Unternehmenspartnern des Projekts erfolgte in $15 \%$ der Fälle, was dem Durchschnitt im Zeitraum 2014-2018 entspricht. Der Wissenstransfer zwischen Wissenschaft und Wirtschaft in Form von Personen umfasste 58 Personen. Wie auch im Vorjahr berichteten $13 \%$ der teilnehmenden FE, dass Mitarbeiter_innen zu anderen F\&E-Projektpartnern wechselten.

Aufgrund des kooperativen Charakters beinahe aller Projektbeteiligungen von FE entstanden in fast $80 \%$ der 
Projektteilnahmen neue Kontakte/Kooperationen (gegenüber $75 \% \mathrm{im}$ Jahr 2017). Diese wurden mit insgesamt 523 neuen Kontakten bzw. 3,2 je Projektteilnahme quantifiziert. Nach dem starken Anstieg auf 4,5 Kontakte durch neun COIN Projektbeteiligungen ist der Wert 2018 wieder gesunken, liegt aber immer noch deutlich über dem Durchschnitt von 2,3 in den Jahren 2014 bis 2016.

\section{Disseminationsaktivitäten erfolgen primär mit Be- teiligung der Projektpartner}

Wie bereits in den Vorjahren ist die Publikation der Projektergebnisse in akademisch referierten Journalen die häufigste Form der Dissemination mit $81 \%$ der rückmeldenden Projektbeteiligungen (Vorjahre: 76-83\%), Konferenzbeiträge ergaben sich in $71 \%$ der Projektbeteiligungen (Vorjahre: $65-73 \%$ ), in $51 \%$ der Nennungen wurden die Ergebnisse in Branchenzeitschriften verbreitet (Vorjahre: 41-50\%). In all diesen Bereichen fand die Dissemination in erster Linie gemeinsam mit Projektpartnern statt, was auf eine relativ enge Kooperation schließen lässt.

Nach Art der FE zeigt sich folgendes Bild: Fachhochschulen und Kompetenzzentren publizierten in $82 \%$ und $85 \%$ der Projektteilnahmen mit ihren Projektpartnern in akademischen Journals und präsentierten die Ergebnisse auf Konferenzen - Journalbeiträge ohne die Projektpartner werden am häufigsten von Forscher_innen an Universitäten und AUF publiziert $(24 \%$ und $15 \%)$. Branchenzeitschriften als Disseminationsmedium werden hingegen häufiger von anwendungsorien- tierteren Forschungseinrichtungen genutzt, insbesondere von Fachhochschulen und Kompetenzzentren. Auch hier erfolgt die Verbreitung vornehmlich mit den Projektpartnern.

Insgesamt wurden 1.531 Transferaktivitäten gesetzt (2017: 1.550), im Durchschnitt 7,3 je Projektteilnahme (Vorjahr: 6,7). Der erneute Anstieg im Vergleich zum Vorjahr kann durch Projekte in den Programmlinien Neue Energien 2020 und einem Projekt im Strukturprogramm Aufbau erklärt werden.

\section{Auch abseits der klassischen Transferaktivitäten von Forschungseinrichtungen wird ein breites Spektrum von Aktivitäten gesetzt}

Neben den „klassischen“ Wegen der Ergebnisverbreitung führten die befragten FE weitere Aktivitäten durch: Etwa die Präsentation bei Veranstaltungen, die Einbindung der Zielgruppe in die Projektdurchführung, bzw. die Zielgruppe des Projektes wurde bei der Umsetzung der Projektergebnisse unterstützt. Im WiMon 2018 wurden das Spektrum möglicher Transferaktivitäten erweitert um Aspekte der "Third Mission“ von Forschung. In diesem Sinne wird erhoben, auf welche Weisen die Projektergebnisse kommuniziert werden, inwiefern die Forschungsergebnisse auch für ein Nicht-Fachpublikum aufbereitet werden, ob auch mit zivilgesellschaftlichen Einrichtungen zusammengearbeitet wird usw.

Die Auskünfte von 187 Projektbeteiligungen werden nach der Art der Forschungseinrichtung in Tabelle 8 dargestellt.

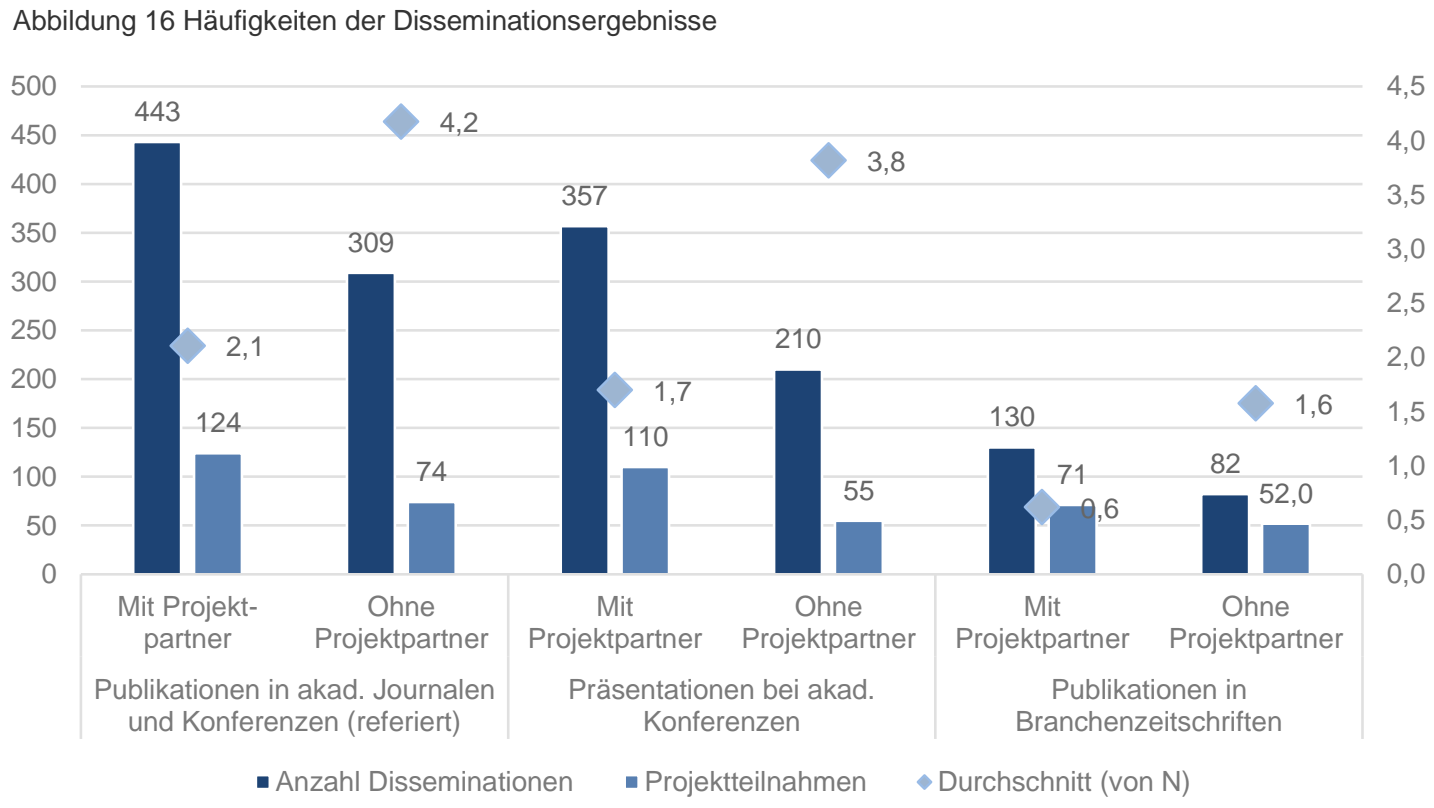

Quelle: $\quad$ KMU Forschung Austria; $n=197$ 
Die Auswertung zeigt leichte Unterschiede zwischen den Arten von Forschungseinrichtungen. Auch wenn sich die Anteile der jeweiligen FE bei den abgefragten Aspekten in einer ähnlichen Größenordnung bewegen, überrascht es, dass sich mehr Außeruniversitäre Forschungseinrichtungen und Fachhochschulen an den Transferaktivitäten beteiligen als Universitäten.

Positiv fällt die relativ hohe Beteiligung von Fachhochschulen in Fachgremien sowie deren hohe Beteiligung an Transferaktivitäten über Medien auf.

Tabelle 8 Aktivitäten zum Transfer der Projektergebnisse

\begin{tabular}{|c|c|c|c|c|}
\hline & AUF & FH & Uni & $\begin{array}{l}\text { Sons- } \\
\text { tige }\end{array}$ \\
\hline $\begin{array}{l}\text { Präsentation der Ergebnisse auf } \\
\text { Fachveranstaltungen }\end{array}$ & $80,3 \%$ & $76,5 \%$ & $74,2 \%$ & $65,2 \%$ \\
\hline $\begin{array}{l}\text { Präsentation der Ergebnisse für } \\
\text { ein nicht-Fachpublikum }\end{array}$ & $40,6 \%$ & $43,8 \%$ & $47,1 \%$ & $26,1 \%$ \\
\hline $\begin{array}{l}\text { Darstellung der Ergebnisse auf } \\
\text { einer Website }\end{array}$ & $81,8 \%$ & $70,6 \%$ & $57,3 \%$ & $82,6 \%$ \\
\hline $\begin{array}{l}\text { Einbindung der Kunden der Pro- } \\
\text { jektpartner }\end{array}$ & $23,8 \%$ & $46,7 \%$ & $28,2 \%$ & $36,4 \%$ \\
\hline $\begin{array}{l}\text { Unterstützung der Projekt- } \\
\text { partner bei der Umsetzung der } \\
\text { Ergebnisse }\end{array}$ & $73,4 \%$ & $87,5 \%$ & $68,2 \%$ & $72,7 \%$ \\
\hline $\begin{array}{l}\text { Beiträge in Printmedien, Social } \\
\text { Media, Blogs, usw. }\end{array}$ & $37,5 \%$ & $58,8 \%$ & $34,5 \%$ & $36,4 \%$ \\
\hline $\begin{array}{l}\text { Kooperation mit zivilgesell- } \\
\text { schaftlichen Einrichtungen }\end{array}$ & $6,3 \%$ & $13,3 \%$ & $11,0 \%$ & $4,5 \%$ \\
\hline $\begin{array}{l}\text { Kooperation mit Bürger_innen } \\
\text { ("Science in Society") }\end{array}$ & $8,1 \%$ & $12,5 \%$ & $12,2 \%$ & $9,1 \%$ \\
\hline $\begin{array}{l}\text { Informationsarbeit für Politik } \\
\text { und Zivilgesellschaft }\end{array}$ & $12,7 \%$ & $25,0 \%$ & $21,4 \%$ & $22,7 \%$ \\
\hline $\begin{array}{l}\text { Mitwirkung in Fachgremien } \\
\text { (Normung, Regulierung, usw.) }\end{array}$ & $18,8 \%$ & $31,3 \%$ & $19,5 \%$ & $13,6 \%$ \\
\hline
\end{tabular}

Quelle: KMU Forschung Austria, N=146. Kategorie „Sonstige“ umfasst Kompetenzzentren, kooperative FE sowie Zentren, Cluster und Netzwerke $(n=41)$

Wirkungen der Projekte primär durch erweiterte Expertise und die Schaffung neuer anwendungsorientierter Fragestellungen

Insgesamt ist die Zustimmung der Forschungseinrichtungen zu den verschiedenen Wirkungsdimensionen mit diesjährigen Wirkungsmonitoring höher als in den Jahren zuvor.

So gestanden $97 \%$ der Befragten dem Projekt zu, die Expertise des Instituts erweitert (Vorjahr: $90 \%$ ) und die Sichtbarkeit der FE erhöht zu haben (83\%, Vorjahr
$80 \%$ ). In $88 \%$ der Fälle ergaben sich aus der Bearbeitung des Projekts neue anwendungsorientierte Fragestellungen (2017: $81 \%$ ), in $60 \%$ auch eher grundlagenforschungsorientierte Fragen für andere Projekte (2017: $56 \%)$. In $49 \%$ der Projektbeteiligungen entstanden neue Lehrinhalte (2017: $42 \%$ ) und in 10 Projektbeteiligungen kam es bereits zu Ausgründungen, in 6 weiteren ist dies zumindest in Planung.

Nach dem Organisationstyp abgegrenzt zeigen sich fallweise durchaus heterogene Wirkungen. So sind GLFnahe Fragestellungen sowie Lehrinhalte eher auf Universitäten zu finden, während AUF die Netzwerkeffekte aufgrund von FFG Projekten besonders hoch einschätzen. 


\section{5 | Verzeichnisse}

\section{Abkürzungsverzeichnis}

$\begin{array}{ll}\text { AUF } & \text { Außeruniversitäre Forschungseinrichtung } \\ \text { DL } & \text { Dienstleistung } \\ \text { EE } & \text { Experimentelle Entwicklung } \\ \text { FE } & \text { Forschungseinrichtung } \\ \text { FH } & \text { Fachhochschule } \\ \text { F\&E } & \text { Forschung und Entwicklung } \\ \text { GLF } & \text { Grundlagenforschung } \\ \text { GU } & \text { Großunternehmen } \\ \text { IF } & \text { Industrielle Forschung } \\ \text { KU } & \text { Kleinunternehmen } \\ \text { KMU } & \text { Kleine und mittlere Unternehmen } \\ \text { MU } & \text { Mittleres Unternehmen } \\ \text { VZÄ } & \text { Vollzeitäquivalent } \\ \text { WiMon } & \text { Wirkungsmonitoring }\end{array}$

\section{Abbildungsverzeichnis}

Abbildung 1 Entwicklung Technologiegruppen seit 2008 (Jahr Projektende): Anzahl Projektbeteiligungen ........ 6

Abbildung 2 Technologieintensität nach Unternehmensgröße ... 6

Abbildung 3 Rolle des FFG-Projekts, Projektende 2007-2014

Abbildung 4 Genderverteilung Projektmitarbeiter Projektende 2007-2014

Abbildung 5 Arten von Innovationen 10

Abbildung 6 Innovationen und deren Kombinationen, 20072014

Abbildung 7 Anmeldung von Schutzrechten in Folge des geförderten F\&E-Projekts, Projektende 20082014

Abbildung 8 Wirtschaftliche Verwertung, Projektende 20112014

Abbildung 9 Zeitpunkt der wirtschaftlichen Verwertung, Einzel- und Kooperationsprojekte..... 13

Abbildung 10 Anteil der gesicherten bzw. zusätzlichen Umsätze bzw. Exporte, die im Ausland erwirtschaftet wurden. Projektende 2008-201413
Abbildung 11 Fördermultiplikator für das Basisprogramm und die übrigen Programme, Projektende 2007-2014

14

Abbildung 12 Additionalität der F\&E-Förderungen. Hätten Sie das Projekt auch ohne Förderung durchgeführt? Projektende 2008-2014.

Abbildung 13 Primäre Rolle des geförderten Projekts im Portfolio der Forschungseinrichtung; Nach Instrumenten..... 16

Abbildung 14 Wie beurteilen Sie die Umsetzungs- und Nutzungsmöglichkeiten der Projektergebnisse?

Abbildung 15 Welche der folgenden Effekte ergaben sich aufgrund des FFG-Projekts? Projektende 20102014.

Abbildung 16 Häufigkeiten der Disseminationsergebnisse. 20

\section{Tabellenverzeichnis}

Tabelle 1 Versendung und Rücklauf: Unternehmen nach Bereichen und Programmen ............................ 5

Tabelle 2 Projektkosten nach Förderungs- und Finanzierungs-instrumenten sowie Unternehmensgröße in Tsd. $€ \ldots \ldots \ldots \ldots \ldots \ldots \ldots \ldots . . . . . . . . . .7$

Tabelle 3 Versendung und Rücklauf: FE nach Bereichen und Programmen

Tabelle 4 Arten der Innovation 10

Tabelle 5 Wirtschaftliche Verwertung der vor vier Jahren abgeschlossenen Projekte, 2018. 12

Tabelle 6 FFG-finanzierte Projektketten, ..................... 17

Tabelle 7 Am Projekt beteiligte F\&E-Mitarbeiter_innen .. 17

Tabelle 8 Aktivitäten zum Transfer der Projektergebnisse 21

Tabelle 9 KMU-Definition der Europäische Kommission 23

Tabelle 10 Zuordnung der Fragebögen zu Programmen und Förderungsinstrumenten der FFG, Unternehmen 23

Tabelle 11 Wurde das Projektziel aus technischer / wirtschaftlicher Sicht erreicht? Bundesländervergleich .. 1

Tabelle 12 Werden die Projektergebnisse im Unternehmen wirtschaftlich verwertet? Bundesländervergleich 


\section{Anhang}

Tabelle 9 KMU-Definition der Europäische Kommission

\begin{tabular}{|c|c|c|c|c|c|}
\hline & $\begin{array}{l}\text { Beschäf- } \\
\text { tigte }\end{array}$ & \multirow{4}{*}{ 号 } & \multicolumn{2}{|c|}{ Umsatz } & $\begin{array}{l}\text { Bilanz- } \\
\text { summe }\end{array}$ \\
\hline $\begin{array}{l}\text { Kleinstun- } \\
\text { ternehmen }\end{array}$ & $<10$ VZÄ & & $\leq 2$ Mio. $€$ & & $\leq 2$ Mio. $€$ \\
\hline $\begin{array}{l}\text { Kleinun- } \\
\text { ternehmen }\end{array}$ & $<50$ VZÄ & & $\leq 10$ Mio. $€$ & 䍃 & $\leq 10$ Mio. $€$ \\
\hline $\begin{array}{l}\text { Mittlere } \\
\text { Unterneh- } \\
\text { men }\end{array}$ & $<250$ VZÄ & & $\leq 50$ Mio. $€$ & & $\leq 43$ Mio. $€$ \\
\hline $\begin{array}{l}\text { Großun- } \\
\text { ternehmen }\end{array}$ & \multicolumn{5}{|c|}{ Abweichende Werte bzw. Eigentümerverhältnisse } \\
\hline
\end{tabular}

Quelle: Europäische Kommission

Diese Grenzwerte dürfen auch gemeinsam mit „Partnerunternehmen" bzw. „verbundenen Unternehmen" nicht überschritten werden, die allein oder gemeinsam mit einem oder mehreren verbundenen Unternehmen
$25 \%$ oder mehr des Kapitals oder der Stimmrechte eines anderen Unternehmens halten.

In der Regel sind KMU eigenständig, d.h., sie sind entweder völlig unabhängig, oder es bestehen Partnerschaften mit anderen Unternehmen mit einer oder mehreren Minderheitsbeteiligungen (von jeweils unter 25\%). Wenn der gehaltene Anteil höher ist, aber 50\% nicht überschreitet, handelt es sich um eine Beziehung zwischen Partnerunternehmen. Liegt er über diesem Schwellenwert, sind die Unternehmen miteinander verbunden.

\section{Beschäftigungseffekt}

$$
\begin{array}{cc} 
& \text { zusätzliche Beschäftigte } \\
+\quad \text { gesicherte Arbeitsplätze } \\
\text { - } \quad \text { freigesetzte Beschäftigte }
\end{array}
$$

\begin{tabular}{|c|c|c|c|c|}
\hline Gruppe & Instrument & Programm & Anzahl & Anteil \\
\hline \multirow{8}{*}{ FEI-Projekt } & C26 Orientierte Grundlagenforschung & NANO & 1 & $0,3 \%$ \\
\hline & C3 E Einzelprojekt EE & Basisprogramm, ASAP & 183 & $46 \%$ \\
\hline & C3-I FEI Einzelprojekt IF & ASAP & 2 & $0,5 \%$ \\
\hline & C4 FEI Kooperationsprojekt EE-IF & $\begin{array}{l}\text { Neue Energien 2020, IV2Splus, FIT-IT, benefit, } \\
\text { ASAP, Produktion der Zukunft, TAKE OFF, } \\
\text { ENERGIE DER ZUKUNFT, COIN, NANO, Ener- } \\
\text { gieforschung }\end{array}$ & 144 & $37 \%$ \\
\hline & C4-G FEI Kooperationsprojekt GLF & Neue Energien 2020 & 2 & $0,5 \%$ \\
\hline & $\begin{array}{l}\text { C4_T FEI Kooperationsprojekt EE od. IF } \\
\text { transnational }\end{array}$ & EUROSTARS, NANO & 3 & $0,8 \%$ \\
\hline & C5 Leitprojekt & $\begin{array}{l}\text { Leuchttürme eMobilität, Neue Energien 2020, } \\
\text { ENERGIE DER ZUKUNFT }\end{array}$ & 24 & $6,2 \%$ \\
\hline & C6 Wissenschaftstransfer & BRIDGE & 14 & $3 \%$ \\
\hline $\begin{array}{l}\text { Markteinfüh- } \\
\text { rung }\end{array}$ & C7 IT & AT:net & 5 & $1,3 \%$ \\
\hline \multirow{2}{*}{ Struktur } & C9 ST Strukturaufbau-Studio & wfForte & 1 & $0,3 \%$ \\
\hline & C10 Innovationsnetzwerk & COIN & 16 & $4 \%$ \\
\hline & & & 390 & $100 \%$ \\
\hline
\end{tabular}

Direkter Beschäftigungseffekt

Tabelle 10 Zuordnung der Fragebögen zu Programmen und Förderungsinstrumenten der FFG, Unternehmen

Quelle: FFG und KMU Forschung Austria 
KMU Forschung Austria
Austrian Institute for SME Research

Tabelle 11 Wurde das Projektziel aus technischer / wirtschaftlicher Sicht erreicht? Bundesländervergleich

\begin{tabular}{|c|c|c|c|c|c|c|c|c|c|c|c|c|c|c|c|c|c|c|c|c|}
\hline & \multicolumn{2}{|c|}{ Gesamtsumme } & \multicolumn{2}{|c|}{ Burgenland } & \multicolumn{2}{|c|}{ Kärnten } & \multicolumn{2}{|c|}{$\begin{array}{l}\text { Niederöster- } \\
\text { reich }\end{array}$} & \multicolumn{2}{|c|}{$\begin{array}{l}\text { Oberöster- } \\
\text { reich }\end{array}$} & \multicolumn{2}{|c|}{ Salzburg } & \multicolumn{2}{|c|}{ Steiermark } & \multicolumn{2}{|c|}{ Tirol } & \multicolumn{2}{|c|}{ Vorarlberg } & \multicolumn{2}{|c|}{ Wien } \\
\hline & abs. & in $\%$ & abs. & in $\%$ & abs. & in $\%$ & abs. & in $\%$ & abs. & in\% & abs. & in $\%$ & abs. & in $\%$ & abs. & in\% & abs. & in\% & abs. & in $\%$ \\
\hline $\begin{array}{l}\text { Projekte ins- } \\
\text { gesamt }\end{array}$ & 390 & $100 \%$ & 11 & $2,8 \%$ & 20 & $5,1 \%$ & 39 & $10,0 \%$ & 105 & $26,9 \%$ & 15 & $3,8 \%$ & 70 & $17,9 \%$ & 25 & $6,4 \%$ & 12 & $3,1 \%$ & 93 & $23,8 \%$ \\
\hline $\begin{array}{l}\text { Gesamtzu- } \\
\text { schuss }^{*} \text { ) }\end{array}$ & 107,6 & $100 \%$ & 3,0 & $2,8 \%$ & 9,7 & $9,0 \%$ & 8,5 & $7,9 \%$ & 24,5 & $22,8 \%$ & 4,6 & $4,3 \%$ & 17,1 & $15,9 \%$ & 9,7 & $9,0 \%$ & 3,2 & $3,0 \%$ & 27,3 & $25,3 \%$ \\
\hline Darlehen $^{*}$ ) & 32,3 & $100 \%$ & 0,7 & $2,2 \%$ & 2,3 & $7,1 \%$ & 1,6 & $5,0 \%$ & 6,7 & $20,7 \%$ & 2,3 & $7,1 \%$ & 3,5 & $10,8 \%$ & 3,4 & $10,6 \%$ & 1,6 & $4,9 \%$ & 10,2 & $31,6 \%$ \\
\hline $\begin{array}{l}\text { technischer } \\
\text { Sicht }\end{array}$ & 356 & $91 \%$ & 11 & $3,1 \%$ & 19 & $5,3 \%$ & 37 & $10,4 \%$ & 96 & $27,0 \%$ & 12 & $3,4 \%$ & 65 & $18,3 \%$ & 21 & $5,9 \%$ & 11 & $3,1 \%$ & 84 & $23,6 \%$ \\
\hline $\begin{array}{l}\text { Gesamtzu- } \\
\text { schuss* }^{*}\end{array}$ & 96,1 & $89 \%$ & 3,0 & $3,1 \%$ & 9,6 & $10,0 \%$ & 8,3 & $8,6 \%$ & 22,3 & $23,2 \%$ & 4,2 & $4,4 \%$ & 15,8 & $16,4 \%$ & 6,2 & $6,5 \%$ & 3,2 & $3,3 \%$ & 23,5 & $24,5 \%$ \\
\hline Darlehen $^{\star}$ ) & 27,2 & $84 \%$ & 0,7 & $2,7 \%$ & 2,2 & $8,3 \%$ & 1,6 & $5,8 \%$ & 5,7 & $21,1 \%$ & 2,1 & $7,8 \%$ & 3,1 & $11,4 \%$ & 1,4 & $5,2 \%$ & 1,6 & $5,8 \%$ & 8,7 & $32,1 \%$ \\
\hline $\begin{array}{l}\text { wirtschaftli- } \\
\text { cher Sicht }\end{array}$ & 242 & $62 \%$ & 7 & $2,9 \%$ & 8 & $3,3 \%$ & 23 & $9,5 \%$ & 65 & $26,9 \%$ & 13 & $5,4 \%$ & 48 & $19,8 \%$ & 11 & $4,5 \%$ & 8 & $3,3 \%$ & 59 & $24,4 \%$ \\
\hline $\begin{array}{l}\text { Gesamtzu- } \\
\text { schuss* }^{*} \text { ) }\end{array}$ & 67,9 & $63 \%$ & 2,3 & $3,4 \%$ & 7,2 & $10,6 \%$ & 6,6 & $9,7 \%$ & 16,6 & $24,4 \%$ & 4,4 & $6,5 \%$ & 11,6 & $17,2 \%$ & 2,3 & $3,4 \%$ & 2,9 & $4,2 \%$ & 14,0 & $20,6 \%$ \\
\hline Darlehen*) & 18,9 & $58 \%$ & 0,5 & $2,8 \%$ & 1,7 & $9,1 \%$ & 1,1 & $6,0 \%$ & 4,1 & $21,7 \%$ & 2,3 & $12,1 \%$ & 2,8 & $15,0 \%$ & 0,9 & $4,6 \%$ & 1,5 & $8,2 \%$ & 3,9 & $20,5 \%$ \\
\hline
\end{tabular}

*) Beträge in Millionen Euro

Inklusive Projektteilnahmen ohne Verwertungsziel 
Tabelle 12 Werden die Projektergebnisse im Unternehmen wirtschaftlich verwertet? Bundesländervergleich

\begin{tabular}{|c|c|c|c|c|c|c|c|c|c|c|c|c|c|c|c|c|c|c|c|c|}
\hline & \multicolumn{2}{|c|}{ Gesamtsumme } & \multicolumn{2}{|c|}{ Burgenland } & \multicolumn{2}{|c|}{ Kärnten } & \multicolumn{2}{|c|}{$\begin{array}{l}\text { Niederöster- } \\
\text { reich }\end{array}$} & \multicolumn{2}{|c|}{$\begin{array}{c}\text { Oberöster- } \\
\text { reich }\end{array}$} & \multicolumn{2}{|c|}{ Salzburg } & \multicolumn{2}{|c|}{ Steiermark } & \multicolumn{2}{|c|}{ Tirol } & \multicolumn{2}{|c|}{ Vorarlberg } & \multicolumn{2}{|c|}{ Wien } \\
\hline & abs. & in\% & abs. & in $\%$ & abs. & in\% & abs. & in $\%$ & abs. & in\% & abs. & in\% & abs. & in\% & abs. & in\% & abs. & in $\%$ & abs. & in\% \\
\hline Projekte insgesamt & 390 & $100 \%$ & 11 & $2,8 \%$ & 20 & $5,1 \%$ & 39 & $10,0 \%$ & 105 & $26,9 \%$ & 15 & $3,8 \%$ & 70 & $17,9 \%$ & 25 & $6,4 \%$ & 12 & $3,1 \%$ & 93 & $23,8 \%$ \\
\hline 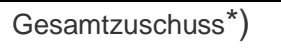 & 107,6 & $100 \%$ & 3,0 & $2,8 \%$ & 9,7 & $9,0 \%$ & 8,5 & $7,9 \%$ & 24,5 & $22,8 \%$ & 4,6 & $4,3 \%$ & 17,1 & $15,9 \%$ & 9,7 & $9,0 \%$ & 3,2 & $3,0 \%$ & 27,3 & $25,3 \%$ \\
\hline Darlehen $^{\star}$ ) & 32,3 & $100 \%$ & 0,7 & $2,2 \%$ & 2,3 & $7,1 \%$ & 1,6 & $5,0 \%$ & 6,7 & $20,7 \%$ & 2,3 & $7,1 \%$ & 3,5 & $10,8 \%$ & 3,4 & $10,6 \%$ & 1,6 & $4,9 \%$ & 10,2 & $31,6 \%$ \\
\hline $\begin{array}{l}\text { werden wirtsch. } \\
\text { verwertet }\end{array}$ & 186 & $48 \%$ & 8 & $4,3 \%$ & 6 & $3,2 \%$ & 21 & $11,3 \%$ & 57 & $30,6 \%$ & 7 & $3,8 \%$ & 35 & $18,8 \%$ & 14 & $7,5 \%$ & 7 & $3,8 \%$ & 31 & $16,7 \%$ \\
\hline Gesamtzuschuss ${ }^{\star}$ ) & 54,7 & $51 \%$ & 2,6 & $4,7 \%$ & 3,7 & $6,7 \%$ & 6,4 & $11,8 \%$ & 15,0 & $27,4 \%$ & 2,7 & $4,9 \%$ & 9,3 & $17,0 \%$ & 3,8 & $7,0 \%$ & 2,8 & $5,2 \%$ & 8,3 & $15,2 \%$ \\
\hline Darlehen*) & 17,4 & $54 \%$ & 0,7 & $4,2 \%$ & 0,2 & $0,9 \%$ & 1,1 & $6,5 \%$ & 4,9 & $28,3 \%$ & 1,4 & $8,3 \%$ & 2,9 & $16,4 \%$ & 1,8 & $10,2 \%$ & 1,5 & $8,9 \%$ & 2,8 & $16,4 \%$ \\
\hline $\begin{array}{l}\text { in Zukunft wirt- } \\
\text { schaftl. verwertet }\end{array}$ & 67 & $17 \%$ & 1 & $1,5 \%$ & 5 & $7,5 \%$ & 5 & $7,5 \%$ & 16 & $23,9 \%$ & 4 & $6,0 \%$ & 14 & $20,9 \%$ & 4 & $6,0 \%$ & 2 & $3,0 \%$ & 16 & $23,9 \%$ \\
\hline Gesamtzuschuss ${ }^{\star}$ ) & 0,0 & $24 \%$ & 0,2 & $0,6 \%$ & 4,0 & $15,6 \%$ & 0,4 & $1,7 \%$ & 3,8 & $14,7 \%$ & 1,2 & $4,9 \%$ & 4,4 & $17,3 \%$ & 2,5 & $9,9 \%$ & 0,2 & $0,6 \%$ & 8,9 & $34,7 \%$ \\
\hline Darlehen*) & 0,0 & $24 \%$ & 0,0 & $0,0 \%$ & 1,6 & $21,3 \%$ & 0,2 & $2,3 \%$ & 0,9 & $11,0 \%$ & 0,6 & $7,8 \%$ & 0,2 & $2,5 \%$ & 0,4 & $5,8 \%$ & 0,0 & $0,0 \%$ & 3,8 & $49,3 \%$ \\
\hline $\begin{array}{l}\text { Erkenntniserweite- } \\
\text { rung }\end{array}$ & 82 & $21 \%$ & 1 & $1,2 \%$ & 6 & $7,3 \%$ & 9 & $11,0 \%$ & 20 & $24,4 \%$ & 4 & $4,9 \%$ & 12 & $14,6 \%$ & 5 & $6,1 \%$ & 1 & $1,2 \%$ & 24 & $29,3 \%$ \\
\hline Gesamtzuschuss ${ }^{*}$ ) & 12,2 & $11 \%$ & 0,2 & $1,5 \%$ & 1,0 & $8,5 \%$ & 1,1 & $8,8 \%$ & 3,8 & $30,9 \%$ & 0,7 & $5,8 \%$ & 1,4 & $11,3 \%$ & 1,8 & $14,5 \%$ & 0,1 & $0,8 \%$ & 2,2 & $18,0 \%$ \\
\hline 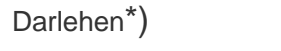 & 2,0 & $6 \%$ & 0,0 & $0,0 \%$ & 0,2 & $8,3 \%$ & 0,2 & $9,4 \%$ & 0,7 & $36,5 \%$ & 0,2 & $12,1 \%$ & 0,1 & $6,5 \%$ & 0,3 & $17,3 \%$ & 0,0 & $0,0 \%$ & 0,2 & $10,0 \%$ \\
\hline keine Verwertung & 13 & $3 \%$ & 0 & $0,0 \%$ & 0 & $0,0 \%$ & 2 & $15,4 \%$ & 2 & $15,4 \%$ & 0 & $0,0 \%$ & 1 & $7,7 \%$ & 1 & $7,7 \%$ & 2 & $15,4 \%$ & 5 & $38,5 \%$ \\
\hline Gesamtzuschuss ${ }^{*}$ ) & 4,4 & $4 \%$ & 0,0 & $0,0 \%$ & 0,0 & $0,0 \%$ & 0,3 & $6,2 \%$ & 0,4 & $8,5 \%$ & 0,0 & $0,0 \%$ & 0,0 & $0,7 \%$ & 0,2 & $4,3 \%$ & 0,1 & $2,6 \%$ & 3,4 & $77,7 \%$ \\
\hline Darlehen $^{*}$ ) & 2,1 & $2 \%$ & 0,0 & $0,0 \%$ & 0,0 & $0,0 \%$ & 0,1 & $5,6 \%$ & 0,0 & $0,0 \%$ & 0,0 & $0,0 \%$ & 0,0 & $0,0 \%$ & 0,1 & $4,3 \%$ & 0,0 & $1,5 \%$ & 1,9 & $88,6 \%$ \\
\hline $\begin{array}{l}\text { kein Verwertungs- } \\
\text { ziel }\end{array}$ & 24 & $74 \%$ & 1 & $4,2 \%$ & 3 & $12,5 \%$ & 2 & $8,3 \%$ & 7 & $29,2 \%$ & 0 & $0,0 \%$ & 6 & $25,0 \%$ & 0 & $0,0 \%$ & 0 & $0,0 \%$ & 5 & $20,8 \%$ \\
\hline Gesamtzuschuss ${ }^{\star}$ ) & 3,4 & $3 \%$ & 0,1 & $1,8 \%$ & 1,0 & $28,5 \%$ & 0,2 & $6,9 \%$ & 0,9 & $26,1 \%$ & 0,0 & $0,0 \%$ & 0,9 & $27,2 \%$ & 0,0 & $0,0 \%$ & 0,0 & $0,0 \%$ & 0,3 & $9,5 \%$ \\
\hline Darlehen*) & 0,3 & $1 \%$ & 0,0 & $0,0 \%$ & 0,3 & $\begin{array}{r}100,0 \\
\%\end{array}$ & 0,0 & $0,0 \%$ & 0,0 & $0,0 \%$ & 0,0 & $0,0 \%$ & 0,0 & $0,0 \%$ & 0,0 & $0,0 \%$ & 0,0 & $0,0 \%$ & 0,0 & $0,0 \%$ \\
\hline
\end{tabular}

*) Beträge in Millionen Euro

Inklusive Projektteilnahmen ohne Verwertungsziel 
